\title{
Feições Oceanográficas em Imagens MODIS na Condição de Sunglint: Exemplos para a Costa Sudeste Brasileira
}

\author{
Lucas Barbedo de Freitas ${ }^{1}$, Milton Kampel ${ }^{1}$, João Antônio Lorenzzetti ${ }^{1}$ \\ ${ }^{1}$ Departamento de Sensoriamento Remoto, Instituto Nacional de Pesquisas Espaciais, \\ São José dos Campos, SP, Brasil.
}

Recebido em 22 de Setembro de 2016 - Aceito em 2 de Abril de 2017

\begin{abstract}
Resumo
Imagens ópticas e no infravermelho-próximo adquiridas por sensores remotos em condição de reflexão especular bidirecional entre o sol, a superfície do mar e o sensor (condição de sunglint) podem ser utilizadas para identificar filmes, feições, processos oceanográficos e meteorológicos atuantes sobre a superfície marinha. Imagens do sensor orbital Moderate Resolution Imaging Spectroradiometer (MODIS) em condição de sunglint na plataforma continental sudeste brasileira foram processadas e analisadas, permitindo identificar feições como a borda Oeste da Corrente do Brasil, meandramentos e vórtices de mesoescala, ondas internas solitárias, rolos atmosféricos e filmes superficiais de origem biogênica e mineral. Uma correção atmosférica mais apropriada para detecção de feições no sunglint é aprensentada, assim como, detalhes sobre a geometria de aquisição. A dependência geométrica do sunglint torna sua ocorrência mais favorável entre outubro e fevereiro na região de estudo quando ocorrem zênites solares mais propícios. Os mecanismos e processos que permitem identificar estas feições no sunglint foram descritos e ilustrados. A partir deste entendimento, as complexas feições identificadas podem ser interpretadas e aplicadas na oceanografia operacional e detecção de vazamentos de óleo na superfície do mar, bem como, para melhorar nosso entendimento sobre os processos físicos e biológicos do meio marinho.
\end{abstract}

Palavras-chave: reflexo do disco solar na superfície do mar, rugosidade da superfície do mar, filmes minerais e biogênicos, ondas internas solitárias, interação oceano-atmosfera.

\section{Oceanographic Features Detected on MODIS Images Under Sunglint Condition: Examples at the Brazilian Southeastern Coast}

\begin{abstract}
Optical/near-infrared remote sensing images acquired by remote sensors in specular bidirectional reflection conditions between sun, sea surface and sensor (sunglint condition) can be used to identify oceanographic and meteorological features and processes at work on the surface of the sea at the time of image acquisition. Moderate Resolution Imaging Spectroradiometer (MODIS) images in sunglint conditions over the Brazilian Southeastern continental shelf were digitally processed and analyzed allowing identify features as the Brazil Current front, mesoscale eddies and meanders, internal solitary waves, atmospheric rolls, biogenic and mineral surface slicks. Base on the understanding about these ocean and atmospheric process and the mechanism which allow us to distinguish these features in sunglint contaminated images. As highlighted here, it is possible to do a better and more detailed interpretation of complex features visible in such images, information that can be applied in operational oceanography, as well as to obtain a better understanding of marine environment processes. The atmospheric correction more suitable to sunglint conditions are here descripted too. As well, the sunglint condition dependence of favorable solar zenith angles which partially restrict its geometry of acquisition (sun, sensor and sea surface) between October and February at the Brazilian Southeastern coast.
\end{abstract}

Keywords: solar disk reflected by the ocean, sea surface roughness, biogenic and mineral films, internal solitary waves, air-sea interactions.

Autor de correspondência: Lucas Barbedo de Freitas, lucasbarbedo@gmail.com.

Present address: Département de Biologie, Chimie et Géographie. Université du Québec à Rimouski, Rimouski, Québec, G5L 3A1, Canada. 


\section{Introdução}

A superfície do mar responde a diversos processos oceanográficos e de interação oceano-atmosfera, produzindo feições específicas de diferentes formas, temperaturas, colorações, com alterações em seu campo de agitação marítima (ondas de superfície) ou em sua rugosidade de pequena escala (ondas curtas de gravidade/capilares) responsáveis pelo sinal de sunglint. O termo sunglint ou Sun glitter é usado para denominar a condição geométrica específica de aquisição de imagens que ocorre de modo especular entre o sensor remoto, a superfície do mar e o sol, quando para um pixel ou uma região da superfície do mar, a luz solar direta é refletida na direção de visada do satélite. Considerando que a superfície do mar não é totalmente lisa e que apresenta um padrão aleatório de oscilação de pequenas facetas que inclinam a superfície, a condição de sunglint não se dá em um único ponto, mas é estendida em uma região de formato elíptico cujo tamanho e brilho é dependente do vento local (Cox e Munk, 1954; Zhang e Wang, 2010). O padrão de rugosidade que modula o sunglint é função do vento, porém é também alterado por processos oceanográficos ou pela presença de filmes de óleo. Assim, a análise do sunglint pode ser aplicada para a estimativa de parâmetros como o vento em superfície ou a identificação de feições oceanográficas (Jackson e Alpers, 2010).

O uso de imagens orbitais do oceano na condição geométrica de sunglint para a caracterização de feições não é uma novidade. Entre as primeiras aplicações desta natureza podemos citar a identificação de feições oceanográficas de mesoescala na costa da Califórnia, a partir de fotografias obtidas do espaço (Soules, 1970). Imagens de satélites meteorológicos da National Oceanic Atmospheric Administration (NOAA), na faixa do visível em condição de sunglint, foram utilizadas para identificar feições de mesoescala associadas a frentes oceanográficas no Atlântico Norte (Violette et al., 1980).

Feições oceanográficas como ressurgências e bordas de correntes podem alterar a instabilidade da camada limite atmosférica e consequentemente o regime de atrito do vento com a superfície do mar. Alterações na dinâmica da coluna d'água também podem imprimir variações na rugosidade superficial. Gagliardini e Colón (2004) mostraram que imagens com sunglint do sensor LANDSAT-Thematic Mapper (TM) são úteis na identificação de vórtices na região da confluência das correntes do Brasil e das Malvinas no Atlântico Sudoeste. Kudryavtsev et al. (2012) utilizaram imagens dos sensores Moderate Resolution Imaging Spectroradiometer (MODIS) e MEdium Resolution Imaging Spectrometer (MERIS) na condição de sunglint para extrair parâmetros dinâmicos da circulação de superfície, derivados da rugosidade da superfície do mar, na região da corrente das Agulhas.

Filmes surfactantes ou tenso-ativos estão associados às substâncias capazes de alterar as caracteristicas fisicas da superficie marinha, como por exemplo, a rugosidade (Espedal et al., 1996). Estas substâncias, dentro de certos limites de turbulência na camada de mistura e velocidade do vento, podem se aglomerar em forma de finas camadas $(\sim 1 \mathrm{~nm})$ sobre a superficie marinha, formando filmes superficiais (Ermakov et al., 1992). A presença de surfactantes minerais e biogênicos tende a forçar um alisamento da superfície pelo efeito de suprimir a formação das ondas capilares. A distribuição de probabilidade das inclinações das facetas das ondas capilares, nas regiões com surfactantes é diferente daquela observada em água limpa. Desta forma, nas regiões em condição de sunglint ocorre um contraste no brilho entre uma área coberta por filmes surfactantes e as áreas adjacentes livres de surfactantes. Este contraste no brilho ocorre mesmo que o filme seja fino e transparente (Hu et al., 2009). Bentz et al. (2004) utilizaram imagens com sunglint do satélite China-Brazil Earth-Resources Satellite (CBERS) para identificar filmes de hidrocarbonetos na superfície do mar, discutindo a utilização dessas imagens em sinergia com outros produtos de sensoriamento remoto para o monitoramento ambiental da costa brasileira. O monitoramento de exsudações naturais de petróleo (Hu et al., 2009) ou de derrames acidentais catastróficos, como o da plataforma Deep Horizon no Golfo do México, também podem ser desenvolvidos com imagens do sensor MODIS em condição de sunglint (Leifer et al., 2012).

A detecção de feições por meio das modulações nas assinaturas da rugosidade da superfície tem sido realizada com a utilização de Radar de Abertura Sintética (SAR, do inglês Synthetic Aperture Radar). A grande vantagem no uso do SAR vem da sua capacidade de adquirir informações da superfície do mar em praticamente quaisquer condições atmosféricas independentemente da iluminação solar (Leifer et al., 2012). O SAR obtém imagens do oceano a partir do sinal retroespalhado na superfície pelas ondas capilares de Bragg em direção à antena do radar (Robinson, 2004). Este aspecto comum de aquisição, determinado por ondas capilares, tanto na condição de sunglint para imagens no espectro visível, quanto em imagens SAR na faixa de micro-ondas, faz com que ambas as técnicas possam ser usadas para detectar as mesmas feições. Entre as feições possíveis de detecção por SAR, podemos mencionar: frentes oceanográficas (Johannessen et al., 1996; Mitnik e Lobanov, 2011), vórtices de mesoescala (Alpers et al., 2013), meandros (Kudryavtsev et al., 2012), ressurgências costeiras (Clemente-Colon, 1999), filmes surfactantes de origem biogênica (Gade et al., 1998), filmes minerais derivados de hidrocarbonetos do petróleo (Leifer et al., 2012), ondas internas solitárias (Lorenzzetti e Dias, 2013), além da possibilidade de se estimar a velocidade do vento (Monaldo et al., 2001) e detectar processos atmosféricos (Topouzelis e Kitsiou, 2015).

O uso de imagens no visível com sunglint requer uma geometria específica de aquisição, além de não poder haver 
cobertura de nuvens. Assim, considerando apenas os aspectos mencionados acima, o sensor SAR apresenta vantagens em relação ao uso das imagens sunglint. Entretanto, enquanto as imagens SAR apresentam custo de aquisição elevado, há um razoável número de sensores orbitais ópticos disponíveis, com suas bases de imagens disponibilizadas gratuitamente para a comunidade científica e o público em geral. Esta maior disponibilidade de imagens deve ser explorada em sinergia com outras técnicas de sensoriamento remoto para aumentar o conhecimento sobre os processos oceanográficos.

Ainda que diversos estudos sobre ondas internas solitarias (OIS) tenham se baseado em imagens SAR (Lorenzzetti e Dias, 2013, entre outros), estas feições também podem ser observadas em imagens de sunglint (Jackson, 2007). As OIS são frequentemente observadas onde as correntes de maré e a estratificação ocorrem próximo a feições topográficas do fundo marinho, como por exemplo, a quebra de plataforma (Apel e Gonzalez, 1983). A passagem de um trem de OIS altera o padrão de convergências e divergências nas correntes próximas à superfície, alterando o padrão de rugosidade marinha e afetando o padrão do sunglint (Pingree e New, 1995). As OIS, da mesma maneira que as ondas de superficie, podem apresentar o fenômeno de arrebentação, promovendo a ressuspensão dos sedimentos de fundo na plataforma continental, trazendo nutrientes para a zona eufótica. Sendo assim, podem ter relevante importância para o incremento na produção primária marinha em ambientes oligotróficos (Muacho et al., 2013; Muacho et al., 2014; Pan et a., 2012). O trabalho de Jackson (2007) utilizou exclusivamente o sensor MODIS na condição de sunglint para o monitoramento global de OIS, demonstrando a aplicabilidade destas imagens em larga escala.

Fenômenos atmosféricos e o atrito do vento alteram os padrões de rugosidade da superfície do mar devido à formação de ondas capilares ou modulações no espectro das ondas capilares. Diversos trabalhos como O'Brien e Mitchell (1988), Wald e Monget (1983), Wylie et al. (1981) utilizaram estas imagens para o cálculo da velocidade do vento próximo à superfície do mar. Khattak et al. (1991) utilizou diferentes bandas do sensor Advanced Very High Resolution Radiometer (AVHRR) adquiridas no mar Mediterrâneo em condição de sunglint para estudar o efeito da Temperatura na Superfície do Mar (TSM) nas variações do vento e rugosidade da camada superficial. A geometria de aquisição e disponibilidade de dados no espectro visível em condição de sunglint permitiram inferir as propriedades de rugosidade da superficie do mar e obter estimativas do campo de vento. A TSM pode ser estimada simultaneamente por bandas no infravermelho termal.

Outro aspecto a ser notado é que a própria condição de reflexão especular do sunglint é normalmente considerada uma limitação ao uso de imagens orbitais para uma série de aplicações oceanográficas. Na condição de glint, a radiância detectada pelo sensor orbital é grandemente dominada pela radiância solar refletida especularmente na superfície do mar, com menor contribuição proveniente dos componentes ópticos-ativos da coluna d'água. Devido a esse efeito, várias agências espaciais mascaram as áreas contaminadas por sunglint nas imagens de cor do oceano para aplicações oceanográficas. Normalmente, no processamento para estimativa da concentração de clorofila na superfície do mar (CSM) por satélite e de outros produtos da cor do oceano, as áreas de sunglint são mascaradas e removidas, ou passam por sofisticadas técnicas de detecção e remoção (Hu, 2011; Kay et al., 2009).

No presente trabalho, apresentamos e discutimos exemplos de aplicação do uso do sunglint para estudos de feições oceanográficas na costa brasileira. Dentre as aplicações, destacam-se a caracterização de feições de mesoescala, a detecção de surfactantes biogênicos e de filmes minerais de origem antrópica, ondas internas solitárias, rolls (rolos) atmosféricos e a estimativa do vento na superfície do mar.

\section{O Sensor MODIS}

O sensor MODIS está a bordo dos satélites Aqua e Terra do programa espacial Earth Observing System (EOS) da National Aeronautics and Space Adminstration (NASA) tendo sido lançados em 1999 e 2002, respectivamente. Estes satélites estão em órbita heliossíncrona, com altitude em torno de $700 \mathrm{~km}$ e campo de visada de $2.330 \mathrm{~km}$, obtendo uma cobertura global diária da superfície terrestre. O sensor MODIS tem 36 bandas dentro do espectro do visível e infravermelho-próximo (NIR). No nadir as bandas centradas em $645 \mathrm{~nm}$ e $859 \mathrm{~nm}$ tem resolução espacial de $250 \mathrm{~m}$ e as demais bandas tem resolução de $500 \mathrm{~m}$ e $1 \mathrm{~km}$. As características de imageamento em conjunto com a larga e ininterrupta série temporal colocam o sensor MODIS como peça chave para o monitoramento e entendimento dos processos do planeta Terra.

A ampla faixa imageada pelo sensor MODIS $(2.330 \mathrm{~km})$ e a ausência de mecanismos para que o sensor varie o ângulo de visada $\theta_{\text {visada }}$ de modo a evitar a geometria de sunglint, faz com que estas feições sejam muito frequentes nas imagens MODIS, formando largas e abrangentes feições elípticas onde o sunglint é processo dominante. esse mascaramento devido ao aumento da intensidade do sunglint, a assinatura do espectro medido pelo sensor possui uma maior influência do espectro solar de acordo com a intensificação do processo de reflexão especular. A Fig. 1, adquirida em 3/2/2014 16:30 GMT, ilustra esse mascaramento, com o aumento da intensidade do sunglint, mais a assinatura do espectro medido pelo sensor se aproximará do espectro solar. 


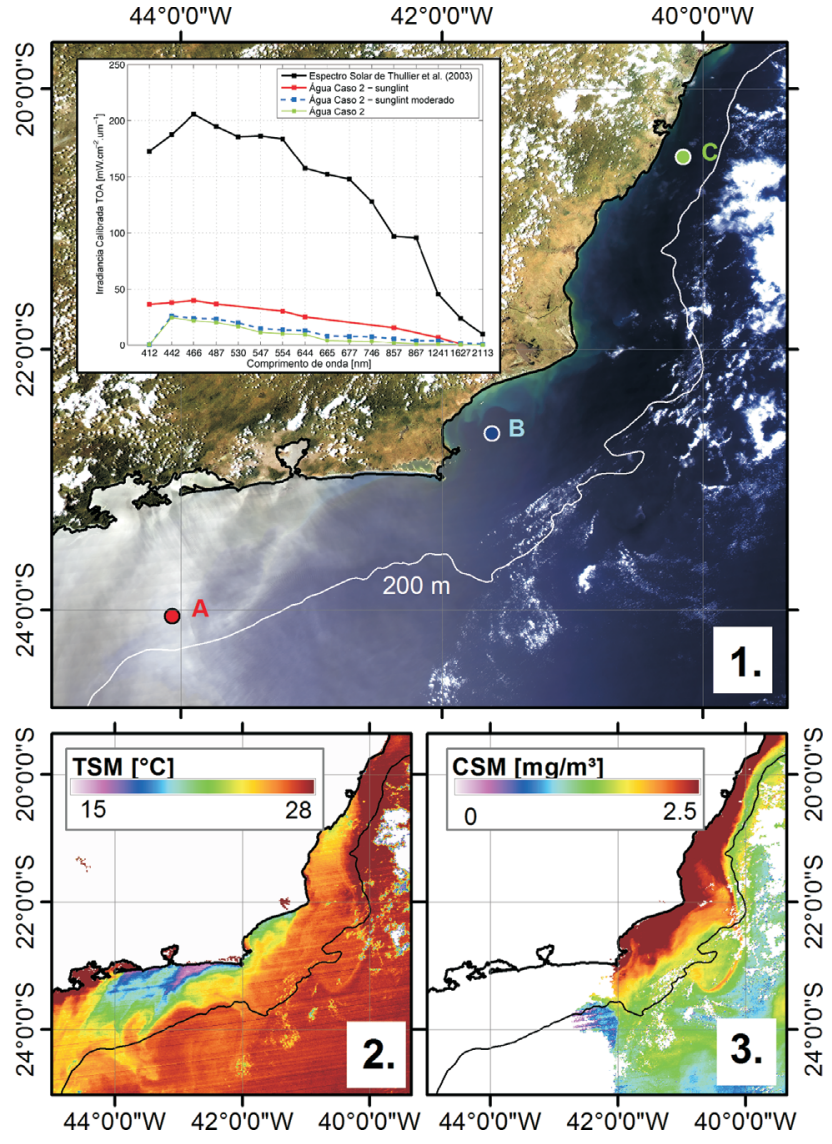

Figura 1 - (1) Imagem Aqua MODIS em composição colorida (R: $645 \mathrm{~nm}, \mathrm{G}: 555 \mathrm{~nm}$ e B: $469 \mathrm{~nm}$ ) com áreas em condição geométrica de sunglint em 3/2/2014 16:45 GMT. Assinaturas espectrais foram adquiridas na condição de intenso sunglint (A), moderado sunglint (B) e fora da condição de sunglint (C) e comparadascom o espectro solar no topo da atmosfera estimado de acordo com Thuillier et al. (2003). (2) Imagem de TSM MODIS com nuvens mascaradas e (3) CSM MODIS com nuvens e sunglint mascarados em branco.

\subsection{Análise das imagens MODIS na condição geométrica de sunglint}

Para descrever e analisar os processos de modulação e formação de ondas capilares, inerentes às principais feições observáveis em áreas de glint, selecionamos um conjunto de feições oceanográficas e meteorológicas julgadas de interesse. Cabendo destacar feições de mesoescala, como vórtices, meandros, frentes da Corrente Brasil, ondas internas solitarias, assinaturas devido a feições atmosfericas, presença de filmes biogênicos e filmes minerais. Um total de 8 imagens descritas na Tabela 1 foram selecioandas a partir do catálogo disponível no site Oceancolor da Nasa, em nível de processamento $1 \mathrm{~B}$, para a região da margem continental brasileira.

As imagens selecionadas foram posteriormente processadas de nivel 1B para o nivel 2, i.e, georreferenciadas e calibradas para radiância, utilizando o programa SeaDAS 7.0.1 (disponivel no site Oceancolor da Nasa). Todas as imagens foram integradas em ambiente de Sistema de In- formações Geograficas (SIG) para facilitar a analise das feições observadas e tambem integra-las a outros parâmetros meteorólogicos e oceanográficos, como a TSM e a concentração de clorofila.

Para melhor caracterização das feições, foi aplicada a técnica de fusão de imagens Gram Schmidt (Karathanassi et al., 2007), utilizando os dados MODIS com resoluções de $500 \mathrm{~m}$ (555 nm e $469 \mathrm{~nm})$ e $250 \mathrm{~m}$ (859 nm e $645 \mathrm{~nm})$, de modo similar ao processamento realizado por $\mathrm{Hu}$ et al. (2009). As bandas MODIS com resolução de $500 \mathrm{~m}$ foram suavizadas através da convolução de filtro mediana com o intuito de minimizar o ruído de faixas (stripes), inerentes à aquisição pushbroom deste sensor. A banda de infravermelho proximo $(859 \mathrm{~nm})$ foi definida como a banda de alta resolução. A principal razão é que a absorção do infravermelho ocorre já na camada de pele da superficie do mar. Assim, a interação com componentes opticamente ativos do interior da coluna d'agua no NIR e quase nula. Via de regra, a quantidade de energia solar refletida no infravermelho proximo, neste caso, deve-se quase exclusivamente à condição de reflexão especular que forma o sunglint

\section{Geometria de aquisção e modelagem do sunglint}

O disco solar pode ser observado refletido na superfície plana e lisa do mar quando existe um ângulo de reflexão especular formado entre o sol, a superfície do mar e um sensor orbital nas bandas do espectro óptico (Ottaviani et al., 2008). Entretanto, a superfície do mar apresenta rugosidade em alta frequência devido às ondas curtas de gravidade/capilar e capilares $(<0,01 \mathrm{~m})$ formadas pelo atrito do vento. A presença de pequenas facetas associadas à rugosidade na superfície do mar produz inclinações em todas as direções, expandindo a área de influência do disco solar refletido. Dentro do grupo de facetas inclinadas aleatoriamente, inclui-se uma fração orientada na direção da reflexão especular do sol para o sensor orbital (Monzon et al., 2006).

Sendo assim, se uma determinada inclinação preferencial das microfacetas estiver no plano de reflexão especular entre o sol e o sensor, haverá um fluxo de energia em direção ao sensor. Portanto, quantificar a energia solar refletida especularmente pela superfície do mar na direção do sensor depende de quantificar a velocidade do vento próximo à superfície e conhecer a geometria de aquisição.

A partir destes conceitos, uma função de densidade de probabilidade $(P D F)$ foi desenvolvida por Cox e Munk (1954) para descrever o comportamento das inclinações das suas microfacetas em função da velocidade e direção do vento in situ, utilizando fotografias aéreas. Bréon e Henriot (2006) e Zhang e Wang (2010) demonstraram que a $P D F$ de Cox e Munk (1954) também pode ser aplicada a imagens de satélite na faixa do visível para quantificar a energia refletida bidirecionalmente na superfície do mar. Zhang e 
Tabela 1 - Descrição das imagens MODIS utilizadas para exemplificar as principais feições observadas em imagens MODIS na condição de sunglint. O vento foi estimado pelos sensores Advanced Scatterometer (ASCAT) da constelação de satélites Polar Orbiting Meteorological Satellites (MetOP) disponibilizados em site do CPTEC/INPE. A direção do vento segue a convenção meteorológica.

\begin{tabular}{|c|c|c|c|c|c|c|}
\hline \multirow[t]{2}{*}{ Satélite } & \multirow[t]{2}{*}{ Dia } & \multirow[t]{2}{*}{ Hora GMT } & \multicolumn{3}{|c|}{ Vento na superfície do mar } & \multirow[t]{2}{*}{ Feições e processos analisados } \\
\hline & & & Mag. m/s & Dir. & Sensor & \\
\hline Aqua & $12 / 11 / 2011$ & $10: 20$ & 2,2 & $220^{\circ}$ & ASCAT & Filmes minerais e sazonalidade do sunglint. \\
\hline Terra & 2/9/2011 & $16: 15$ & 10,0 & $300^{\circ}$ & ASCAT & Foz do Rio Amazonas. \\
\hline Terra & $5 / 1 / 2013$ & $13: 15$ & 4,2 & $145^{\circ}$ & ASCAT & Filmes biogênicos na bacia de Campos. \\
\hline Aqua & $9 / 1 / 2010$ & $16: 25$ & 4,0 & $150^{\circ}$ & ASCAT & Variações na estabilidade da camada limite atmosférica. \\
\hline Aqua & $3 / 2 / 2014$ & $16: 15$ & 3,5 & $225^{\circ}$ & ASCAT & Análise espectral. \\
\hline Aqua & $2 / 1 / 2010$ & $16: 20$ & 10,5 & $120^{\circ}$ & ASCAT & $\begin{array}{l}\text { Borda da Corrente do Brasil formando linha de intensa } \\
\text { convergência. }\end{array}$ \\
\hline Terra & $12 / 2 / 2015$ & $13: 15$ & 9,0 & $120^{\circ}$ & ASCAT & Rolls atmosféricos. \\
\hline Aqua & $6 / 1 / 2013$ & $16: 40$ & 7,0 & $240^{\circ}$ & ASCAT & $\begin{array}{l}\text { Rolls atmosféricos, sombra de nuvens, sólions (ondas internas } \\
\text { solitárias). }\end{array}$ \\
\hline
\end{tabular}

Wang (2010) apresentaram uma versão simplificada da equação original de Cox e Munk (1954) denominada isotrópica por que considera apenas a magnitude do vento e não variações na direção do vento (Eq. (1)):

$$
P D F=\frac{1}{\pi \sigma_{i}^{2}} \exp \left(\frac{-\tan ^{2} \beta}{2 \sigma_{i}^{2}}\right)
$$

sendo $i=$ [água; surfactante], onde $\sigma_{i}^{2}$ é a variância (média quadrática) das inclinações em função da magnitude do vento e da presença ou não de surfactantes $(i)$ e plano- $\beta$ descreve a orientação espacial do plano para reflexão especular entre o sol e o sensor (Eq. (2)):

$$
\tan \beta=\sqrt{Z_{x}^{2}+Z_{y}^{2}}
$$

A Fig. 2 descreve a geometria de aquisição de imagens orbitais de sensores ópticos. A direção do sol determina o eixo-x do sistema, os termos $Z_{x}$ e $Z_{y}$ são as componentes da inclinação do plano- $\beta$ na direção do Sol (eixo- $x$ ) e perpendicular ao azimute solar (eixo-y). $Z_{x}$ e $Z_{y}$ podem ser deduzidos nas Eqs. (3.a,b) pelo zênite solar $\theta_{\text {sol }}$, zênite de visada do sensor $\theta_{\text {visada }}$ e azimute relativo entre o sol e o sensor $\phi$ :

$$
\begin{aligned}
& Z_{x}=\frac{\sin \theta_{\text {sol }}+\sin \theta_{\text {visada }} \cos \phi}{\cos \theta_{\text {sol }}+\cos \theta_{\text {visada }}} \\
& Z_{u}=\frac{\sin \theta_{\text {visada }} \sin \phi}{\cos \theta_{\text {sol }}+\cos \theta_{\text {visada }}}
\end{aligned}
$$

Zhang e Wang (2010) mostram que a $P D F$ anisotrópica de Cox e Munk (1954) que considera a direção e magnitude do vento, alcança melhor desempenho para modelar o sunglint do sensor MODIS. Entretanto, o modelo isotrópico também alcança resultados satisfatórios de forma simplificada. Cabe mencionar que existem diversos modelos e algoritmos publicados na literatura para quanti-

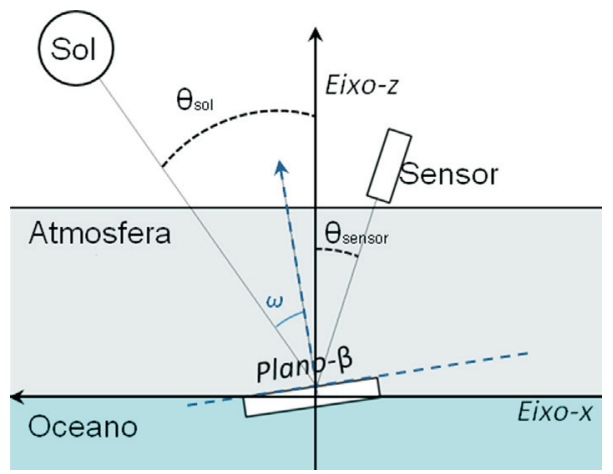

Figura 2 - Esquematica da geometria de aquisição de imagens orbitais de sensores ópticos: sistema sol, superfície do mar e sensor (ver texto para explicação dos termos). O ângulo de azimute relativo ao sol e a visada do sensor $\varphi$ é $180^{\circ}$.

ficar o sunglint. Por exemplo, modelos parametrizados utilizando estimativas de vento por escaterômetros (Bréon e Henriot, 2006), modelos não lineares, entre outros discutidos e analisados por Zhang e Wang (2010).

A concentração de filmes surfactantes torna a superfície do mar menos extensível, dissipando as ondas capilares e suavizando a rugosidade. Consequentemente, este efeito interfere na inclinação das microfacetas e o efeito da velocidade e direção do vento produz inclinações diferentes para condições de superfícies com e sem a presença de surfactantes. Cox e Munk (1954) definiram os valores de $\sigma_{i}^{2}$ da Eq. (1), em função da velocidade do vento $V(\mathrm{~m} / \mathrm{s})$, da presença de surfactantes $\left(\sigma_{\text {surfactante }}^{2}\right)$ ou ausência $\left(\sigma_{\text {agua }}^{2}\right)$. Desta forma, tanto filmes biogênicos (Gower et al., 2006) como filmes minerais (Hu et al., 2009) podem ser detectados em imagens na condição de sunglint (Eqs. (4.a,b)):

$$
\begin{aligned}
& \sigma_{\text {surfactantes }}^{2}=0,008+0,00156 \mathrm{~V} \\
& \sigma_{\text {agua }}^{2}=0,003+0,00512 \mathrm{~V}
\end{aligned}
$$


Considerando a relação direta de $\sigma_{i}^{2} \operatorname{com} V$ (Eqs. (4.a,b)) e a $P D F$ das inclinações (Eq. (1)), ocorre que com o aumento (diminuição) da magnitude do vento tem-se uma diminuição (aumento) do valor de pico da $P D F$ e seu alargamento (estreitamento).

Como descrito por Bréon e Henriot (2006), a reflectância especular que ocorre na superfície do mar, ou seja, a relfectância de sunglint $\left(r_{g}\right)$ (Eq. (5)) considerando a orientação das microfacetas estimadas pela $P D F$ de Cox e Munk (1954) em uma geometria específica de aquisição é dada por:

$$
r_{g}=\frac{\pi I}{E_{o} \cos \theta_{\text {sol }}}=\frac{\pi f(\omega, \lambda)}{4 \cos \theta_{\text {sol }} \cos \theta_{\text {visada }} \cos ^{4} \beta} P D F
$$

sendo $i=$ [surfactante; água], $I$ é a radiância refletida, $E_{0}$ $\cos \theta_{\text {sol }}$ é a irradiância solar considerando a declinação solar. O termo $f(\omega, \lambda)$ é o índice de reflexão de Fresnel em função do comprimento de onda $\lambda$ e ângulo de reflexão $\omega$, descrito abaixo na Eq. (6):

$$
\cos 2 \omega=\cos \theta_{\text {sol }} \cos \theta_{\text {visada }}+\sin \theta_{\text {sol }} \sin \theta_{\text {visada }} \cos \phi
$$

O sinal do sunglint recebido pelo sensor remoto sofre também o efeito da atmosfera. No visível, este efeito ocorre principalmente devido aos gases e aerossóis. Para minimizar esse efeito, modelos de correção atmosférica podem ser aplicados como o descrito por Tanré et al. (1979). Para tal, a profundidade óptica dos aerossóis $\tau_{\text {aer }}$ e molecular $\tau_{m o l}$ são removidas, considerando a trajetória da radiação na atmosfera $\left(1 / \cos \theta_{\text {sol }}+1 / \cos \theta_{\text {visada }}\right)$. Assim, a reflectância de sunglint com a correção atmosférica $\rho_{g}$ aplicada segundo Tanré et al. (1979) pode ser descrita como $\rho_{r}=r_{g} T_{o}$, onde $T_{o}$ é a transmitância atmosférica na trajetória direta da radiação. Usando a relação entre a reflectância de sunglint $\rho_{g}$ e a radiância de sunglint $L_{g}$ temos (Eq. (7)):

$$
\rho_{g}=\frac{\pi L_{g}}{F_{o} \cos \theta_{\text {sol }}}
$$

O mascaramento de áreas de sunglint para estimativas de parâmetros bio-ópticos, como descrito por Wang e Bailey (2000), utiliza o conceito de radiância de sunglint normalizada $\left(L N_{G N}\right)$, definida por $L_{g}=F_{o} T_{o} L N_{G N}$, sendo $F_{o}$ e $T_{o}$ a irradiância solar extraterrestre (ajustada para as variações de distância entre a Terra e o Sol) e a transmitância atmosférica direta na direção do Sol, respectivamente. O valor de $F_{o}$ pode ser quantificado pelo espectro solar de Thuillier de acordo com a banda espectral do sensor orbital (Thuillier et al., 2003). Por exemplo, para a banda MODIS centrada em $645 \mathrm{~nm}$, com resolução espacial de $250 \mathrm{~m}$ e resolução espectral de $10 \mathrm{~nm}$, a irradiância média $F_{o}$ é igual a $158,74 \mathrm{~mW} \mathrm{~cm}^{-2} \mu \mathrm{m}^{-1}$. McClain et al. (1995) usam uma fração do valor $L_{g}(850 \mathrm{~nm})$ para assumir a contaminação por sunglint em imagens ópticas destinadas a estimativas de parâmetros bio-ópticos. Nos sensores Sea-viewing Wide
Field-of-view Sensor (SeaWiFS) e MODIS o limite de $0,005 F_{o}(859 \mathrm{~nm})$ é considerado altamente contaminado. Imagens em condição de fraco sunglint, com frações abaixo do limite 0,005 podem ser corrigidos como descrito para o sensor MERIS por Steinmetz et al. (2011) e para o sensor SeaWiFS por Wang e Bailey (2001).

Mesmo em imagens na condição geométrica de sunglint, parte das orientações das microfacetas não se incluem no ângulo de reflexão bidirecional (sol-superfície-sensor). Sendo assim, parâmetros bio-ópticos também influenciam na quantidade de energia recebida pelo sensor. Imagens nestas condições podem apresentar uma grande variedade de detalhes e alta complexidade. Como exemplo desta variedade de feições, a imagem da foz do rio Amazonas (Fig. 3) adquirida pelo sensor MODIS em 2/9/2006 às 16:15 GMT, os dados de vento estimados pelo National Centers for Environmental Prediction (NCEP) denotam magnitude média do vento de $10 \mathrm{~m} / \mathrm{s}$ e direção de $300^{\circ}$, variando entre 1,5 e $9,3 \mathrm{~m} / \mathrm{s}$ na componente zonal e entre $-1,2$ e $0,9 \mathrm{~m} / \mathrm{s}$ na componente meridional.

\subsection{Sazonalidade da condição de sunglint}

A qualidade das imagens no visível é dependente da geometria de aquisição, ou seja, a relação entre a iluminação solar e a visada do sensor. A órbita heliossíncrona é caracterizada por manter a condição de iluminação solar o mais constante possível ao longo do tempo. Porém, quando a geometria de aquisição se aproxima da geometria de reflexão especular essas imagens tendem a ser contaminadas pela condição de sunglint. Mesmo se considerar uma repetição da órbita, conservando os zênites e azimutes de visada, a declinação solar varia sazonalmente o que resulta

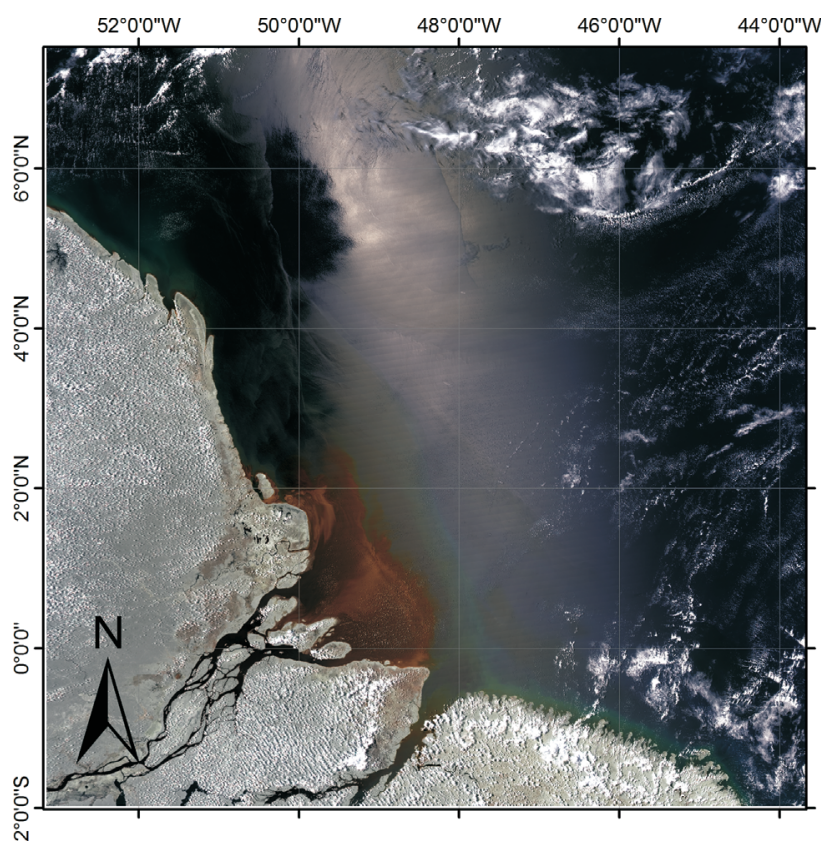

Figura 3 - Imagem MODIS na condição de sunglint sobre a Foz do Rio Amazonas, adquirida dia 2/9/2006 às 16:33GMT. 
na sazonalidade da condição de sunglint, restringindo esta a determinados períodos do ano quando a declinação solar está mais próxima do nadir. Sendo assim, a ocorrência da condição de sunglint nas aquisições MODIS em regiões tropicais e subtropicais da costa do Brasil, possui também uma característica sazonal, confinada entre meados da primavera e início do outono austral.

A ocorrência da condição de sunglint durante o ano pode ser avaliada para determinada posição, considerando as características de visada de cada sensor. Frulla et al. (1995) descrevem as implicações da geometria de iluminação e visada do sensor AVHRR, considerando a ocorrência da contaminação por sunglint a partir do ângulo entre a visada do sensor e o ângulo para reflexão especular, denominado ângulo de cone de sunglint- $\xi$ (Eq. (8)):

$$
\cos \xi=\mu \mu_{o}-(1-\mu)^{\frac{1}{2}}\left(1-\mu_{o}^{2}\right)^{\frac{1}{2}} \cos \phi
$$

sendo $\mu=\cos \theta_{\text {visada }} ; \mu_{o}=\cos \theta_{\text {sol }}$.

A contaminação por sunglint é mais provável de ocorrer quando a geometria de aquisição se aproxima da geometria de reflexão especular. Assim, se o ângulo de cone for menor que o limiar de $15^{\circ}$, podemos considerar a presença de sunglint.

O ângulo de cone é dependente das inclinações das microfacetas da superfície marinha e pode variar de acordo com a intensidade e direção do vento. Em situação de ventos muito fracos que condicionam uma superfície lisa e sem rugosidade, a condição de sunglint estaria restrita apenas à geometria de aquisição, extremamente próxima a reflexão especular. As inclinações das ondas capilares é limitada, o que restringe o ângulo de cone de sunglint mesmo em condições de ventos muito intensos, pois caso contrário, o cone de sunglint poderia se estender até o horizonte (Zeisse, 1994).

Alguns autores selecionam um valor fixo para o cone de sunglint, como o limite de $15^{\circ}$ definido por Frulla et al. (1995) para o sensor AVHRR e também por Kehoe (2002) para sensores geoestacionários. Porém, a definição do ângulo de cone deve ser relacionada ao limite de inclinação das microfacetas, podendo variar em função da velocidade e direção do vento.

Avaliamos a sazonalidade do sunglint a partir de um transecto acompanhando uma linha de varredura do sensor MODIS (plataforma orbital Terra) no embaiamento da costa sudeste brasileira (Fig. 4). Considerando a repetição da órbita heliossíncrona, conservamos o ângulo de visada e azimute de cada pixel do transecto mas alteramos a posição dos ângulos solares a cada mês, sempre às 13:05 GMT. Dentro dos intervalos entre janeiro-fevereiro e outubro-dezembro, os valores da radiância normalizada de sunglint são intensos e acima do limite máximo para a estimativa de parâmetros bio-ópticos, i.e. $L N_{G N}>0,005 \mathrm{sr}^{-1}$ (McClain et al., 1995).

\section{Feições de Mesoescala: Frentes Oceanográficas, Vórtices e Meandros}

Frentes oceanográficas delimitam massas d’água, demarcando diferentes domínios e ambientes de características físico-químicas específicas (Belkin e Miller, 2009). Sobre a plataforma continental sudeste do Brasil, estas frentes delimitam os domínios das seguintes massas d'água (Stramma e England, 1999): Água Costeira (AC), Água Tropical (AT) e Água Central do Atlântico Sul (ACAS). Em algumas regiões, a ACAS aflora na superfície por ressugência costeira, formando frentes térmicas, como descrito por Castelao e Barth (2006) para a região de Cabo Frio, RJ. Bakun (2006) discorre sobre a importância das frentes, como processo de incremento na produção primária devido a transferências de propriedades entre duas diferentes massas d'água, como nutrientes e temperatura.

Outros aspectos relacionados às frentes oceanográficas são a gênese e dinâmica de vórtices e meandros. Feições como vórtices de mesoescala e sub-mesoescala são de grande importância para a sobrevivência de peixes e processos de sucessão ecológica (Rodríguez et al., 2004). Igualmente importantes são as transferências de calor e propriedades por longas distâncias, associadas às características conservativas dos vórtices (Schouten et al., 2000). A formação de meandros pode também, intensificar o processo de ressurgência costeira, dependendo da posição sobre a quebra da plataforma (Calado et al., 2010).

De acordo com Kudryavtsev et al. (2012), as assinaturas no sunglint desses fenômenos permitem descrever a dinâmica local, podendo assim ser aplicadas em apoio às operações marítimas. A capacidade de observar frentes oceanográficas, dentro da condição geométrica de sunglint, é descrita em Violette et al. (1980) para o sensor AVHRR. Entre outros trabalhos mais recentes, podemos citar o uso de imagens pancromáticas estereográficas de fina resolução espacial ( $\sim 2,5 \mathrm{~m}$ ) por Matthews e Awaji (2010). As diferenças de temperatura entre as massas d'água que compõem as frentes oceanográficas podem efetivamente alterar o padrão de rugosidade na superfície do mar (Gagliardini e Colón, 2004). Uma modulação das ondas capilares pode ocorrer por processos dinâmicos como divergência ou convergência na coluna d'água, ou mesmo, por variação na estabilidade da camada limite atmosférica. Este padrão pode ser observado na imagem MODIS de 9/1/2010 16:25 GMT (Fig. 5) pelas variações no brilho devido à diferença de TSM e consequente variação da estabilidade da camada limite. A presença de nuvens também influencia a observação da região oceânica nesta imagem, formando manchas escuras alongadas.

Como descrito por Belkin e O'Reilly (2009) e esquematizado na Fig. 6 no lado mais quente da frente, a ascensão do vapor d'água associado à transferência de calor latente aumenta a turbulência na camada limite. Isto resulta em instabilidade na camada limite e aumento do vento de 


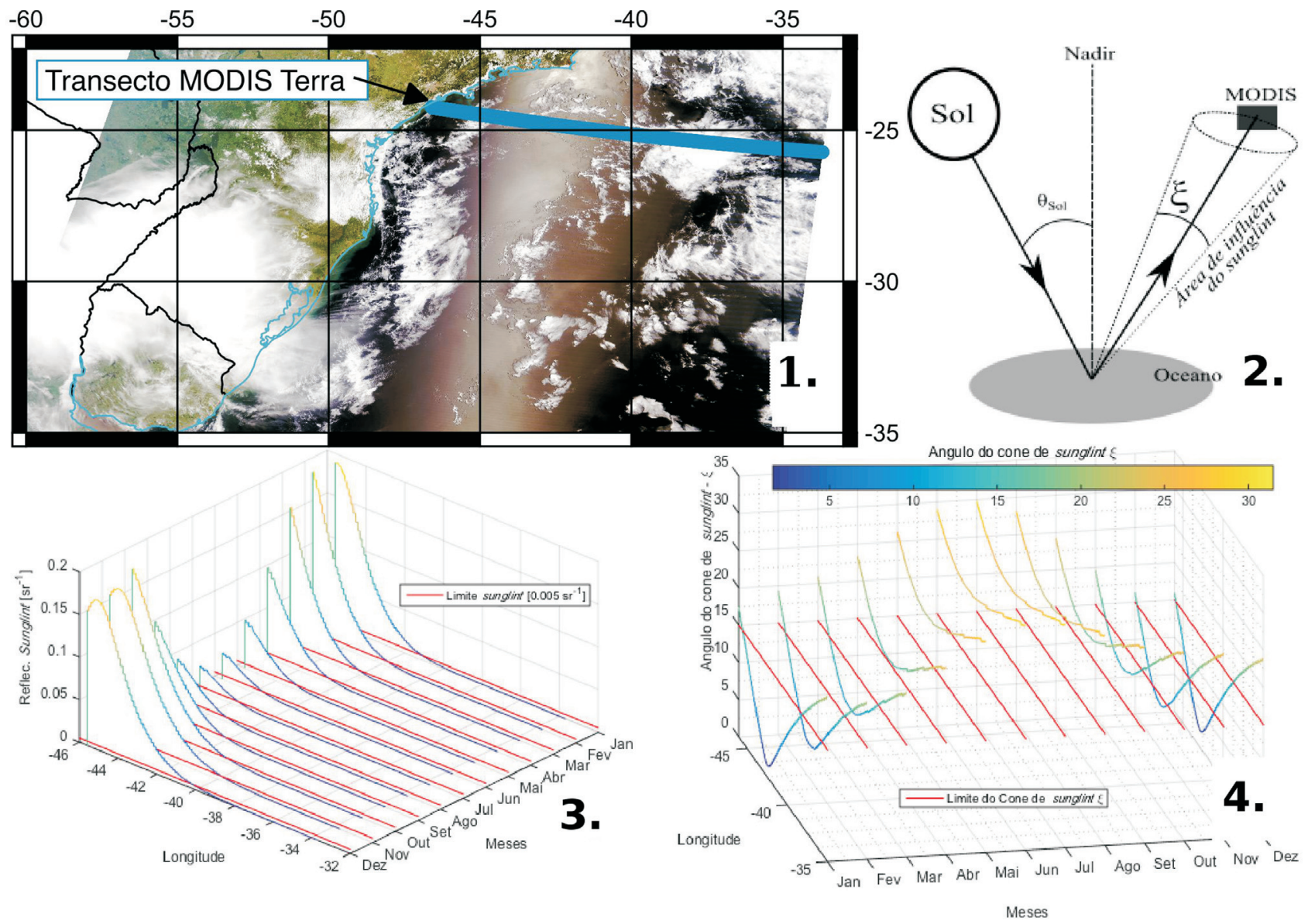

Figura 4 - Sazonalidade da ocorrência de sunglint em imagens do sensor MODIS, satelite Terra. (1). Transecto acompanhando uma faixa de varredura do sensor MODIS, satelite Terra (linha vermelha), em aquisição contemplando o largo do embaiamento de Santos, costa sudeste brasileira e regiões oceanicas, em 5/1/2015 às 13:05 GMT. (2) Esquema da geometria de aquisicao considerando o cone de sunglint- $\xi$ (ver texto). (3) Angulo entre o sensor e o sol para o transecto durante o ano em relacao ao angulo de cone de sunglint- $\xi$. Geometrias dentro do cone, i.e. menores que $15^{\circ}$, são potencialmente contaminadas pelo sunglint. (4) Modelagem da variacao sazonal da relfectancia de sunglint. Conservando a geometria de visada, a variacao da declinacao solar determina a intensidade do sunglint. O limite de $0,005 \mathrm{sr}^{-1}$ (de acordo com McClain et al., 1995) determina a contaminação por sunglint em imagens do espectro visivel.

superfície. Já no lado mais frio da frente, os processos de instabilidade na camada limite são diminuídos, resultando em um vento de superfície menos intenso e, consequentemente, uma suavização na rugosidade da superfície do mar.

A imagem MODIS adquirida na condição de sunglint em 2/1/2010 às 16:20 GMT (Fig. 7) contempla as diferenças de brilho no sunglint causadas pelo contraste de temperatura entre as massas de água $\mathrm{AC}$ e AT. Sob o regime da AT caracterizada por temperaturas $>20{ }^{\circ} \mathrm{C}$ e salinidade $>36,40$ (Campos et al., 2000), em águas quentes da Corrente do Brasil, a camada limite atmosférica tende a ser mais instável que em áreas de $\mathrm{AC}$ relativamente mais frias com temperaturas $>15{ }^{\circ} \mathrm{C}$ e salinidade $<35,0$ (Brandini, 1990). Em áreas de superfície mais quentes a instabilidade forma uma superfície marinha mais rugosa e vice-versa. A análise da imagem MODIS na banda do infravermelho próximo $(859 \mathrm{~nm})$ permite uma melhor discriminação das variações de brilho devidas às variações na estabilidade da camada limite (Fig. 7).

O infravermelho próximo tem mínima penetração na coluna d'água, restrita à camada de pele. Porém, na condição de sunglint grande parte das microfacetas estão em ângulo de reflexão especular de acordo com o coeficiente de Fresnel. Neste caso, a radiação é refletida na superfície. Assim, as assinaturas registradas no infravermelho próximo devem-se principalmente às feições de sunglint e não a processos interiores da coluna d'água. Cabe ressaltar que a condição de sunglint não impede que a radiação eletromagnética no espectro visível penetre na coluna e interaja com seus componentes opticamente ativos uma vez que as microfacetas da superfície tem orientações muito variadas. Desta forma, é possível observar feições da cor do oceano como a faixa de alta concentração de clorofila na região costeira em torno do Cabo de São Tomé, RJ (Fig. 7).

Ainda a respeito da frente térmica entre a AT e AC, ocorre uma linha fortemente demarcada na feição de 


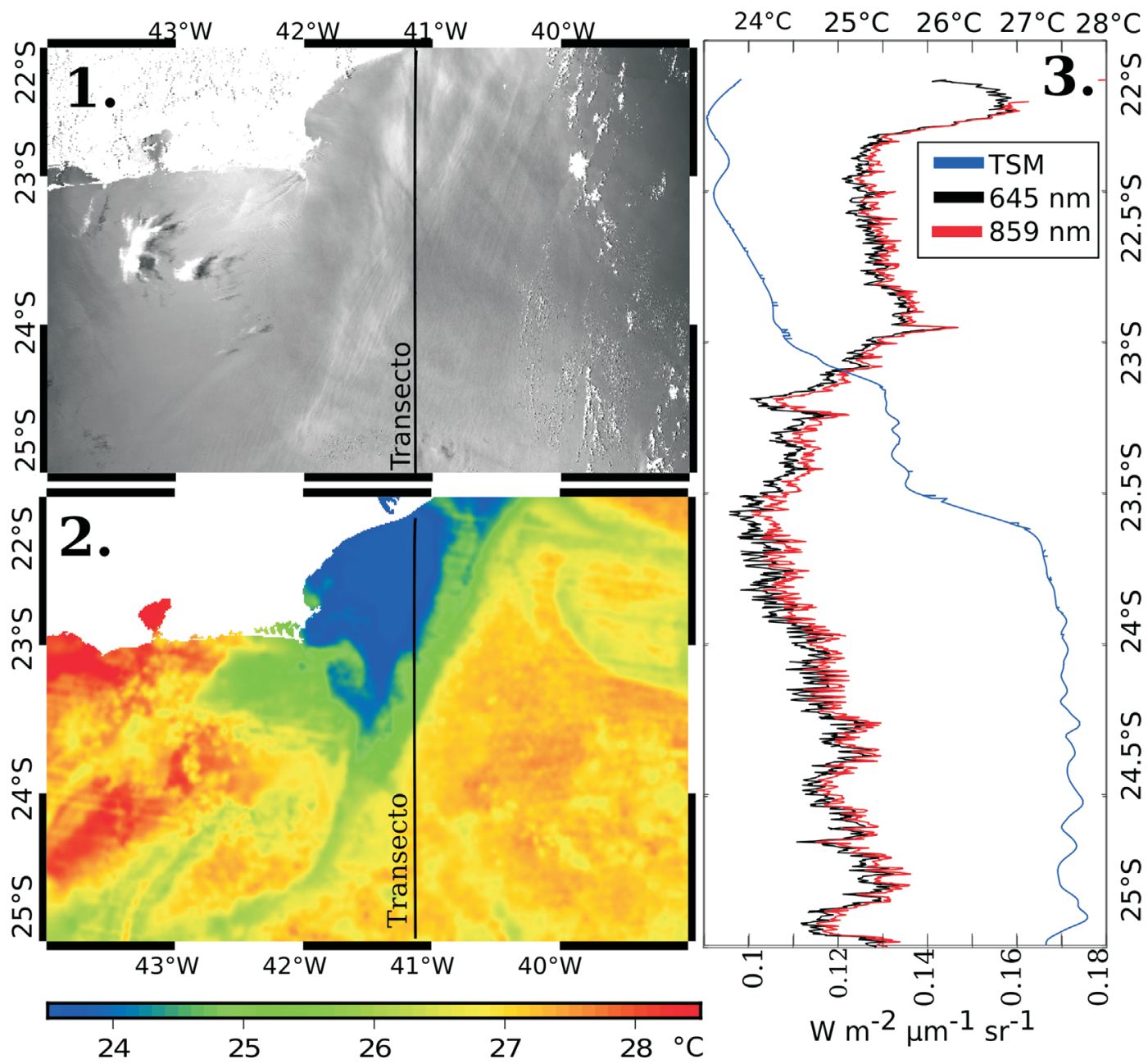

Figura 5 - Imagem do MODIS Aqua (859 nm) na condição de sunglint em 9/1/2010 às 16:25 GMT sobre aplataforma continental sudeste brasileira. Observa-se que variações de brilho na imagem do infravermelho próximo (1) coincidem com feições de TSM (2) devido ao seu efeito na estabilidade da camada limite oceano-atmosfera. (3) O transecto demostra a variação do sunglint, nas bandas do vermelho (645 nm) e infravermelho proximo (859 nm), relacionada com a variação na TSM.Como descrito por Belkin e O'Reilly (2009) e esquematizado na Fig. 6 no lado mais quente da frente, a ascensão do vapor d'água associado à transferência de calor latente aumenta a turbulência na camada limite. Isto resulta em instabilidade na camada limite e aumento do vento de superfície. Já no lado mais frio da frente, os processos de instabilidade na camada limite são diminuídos, resultando em um vento de superfície menos intenso. Consequentemente, uma suavização na rugosidade da superfície do mar.

sunglint coincidindo espacialmente com a borda norte de um vórtice ao largo do Cabo de São Tomé, RJ (Fig. 7). Esta linha pode ser relacionada dinamicamente à forte convergência neste caso associada a um meandro da Corrente do Brasil. Segundo Yoder et al. (1994), este tipo de feição é característico de frentes com intensa convergência, subducção e com acúmulo de biomassa fitoplanctônica devido às trocas de propriedades entre as massas d'água. Na imagem, a aparência de uma pequena elevação no nível do mar deve-se em geral à quebra de ondas pelo vento (whitecaps ou carneirinhos).

\section{Filmes Biogênicos e Minerais}

Filmes surfactantes ou tensoativos estão associados às substâncias capazes de alterar as características físicas da superfície marinha, como por exemplo, a tensão superfi-

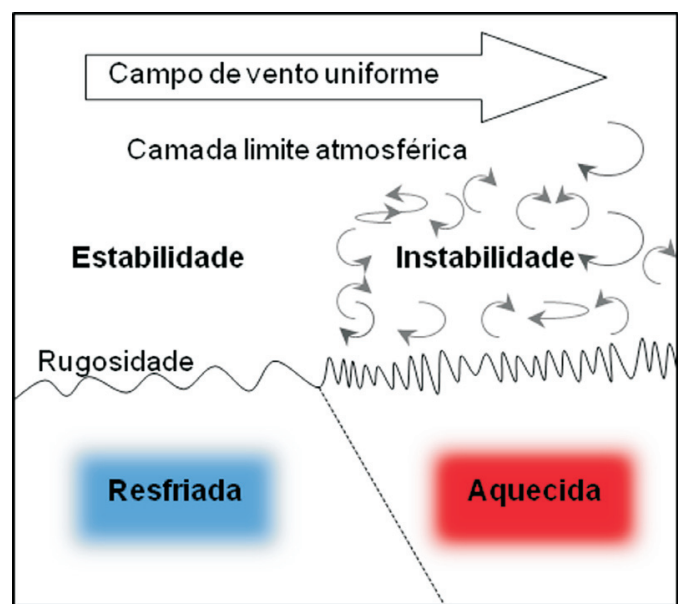

Figura 6 - Esquema ilustrativo da diferente resposta na rugosidade do oceano em função da ocorrência de frentes térmicas. Adaptada de Robinson (2004). 

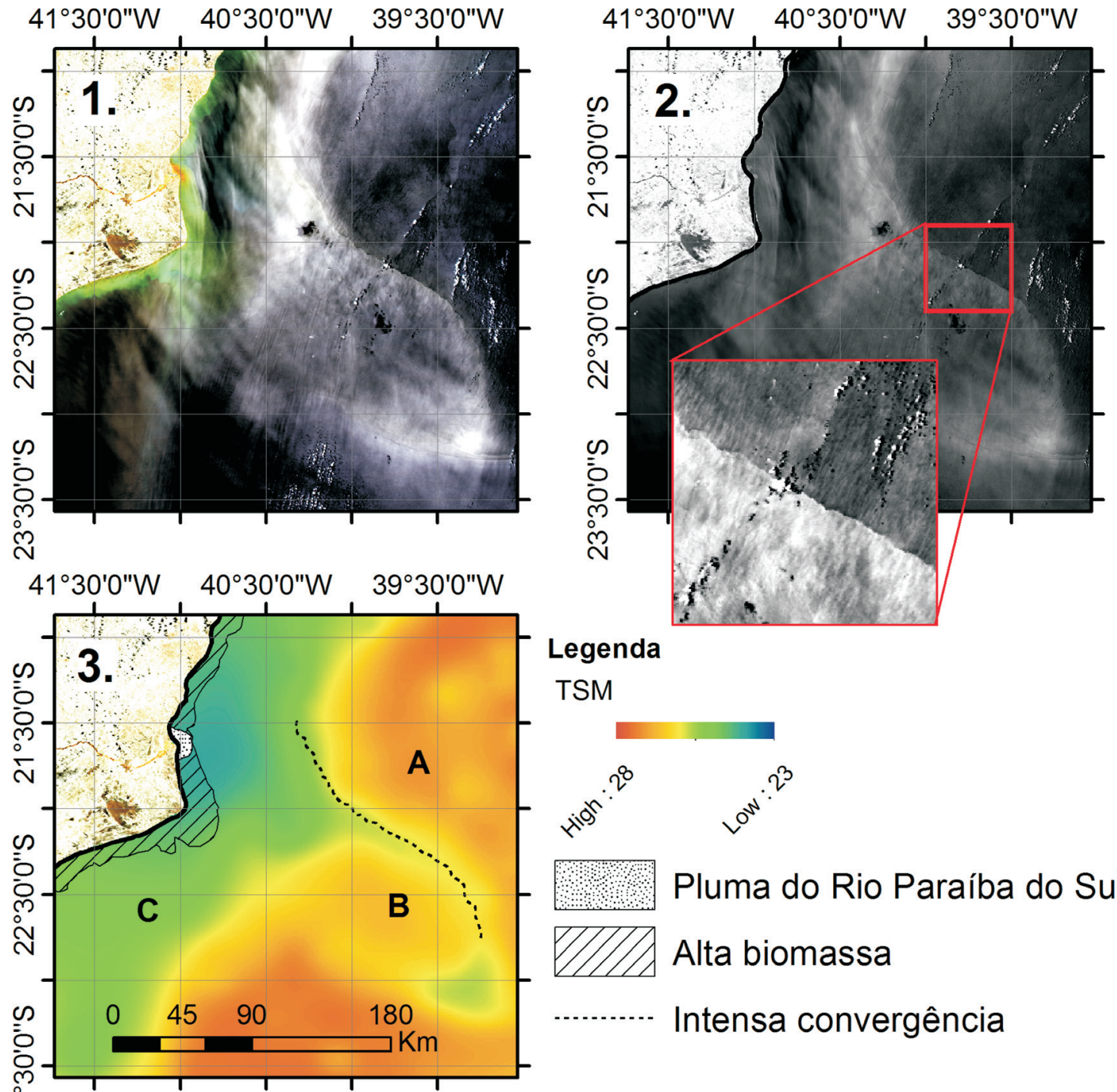

Legenda

TSM

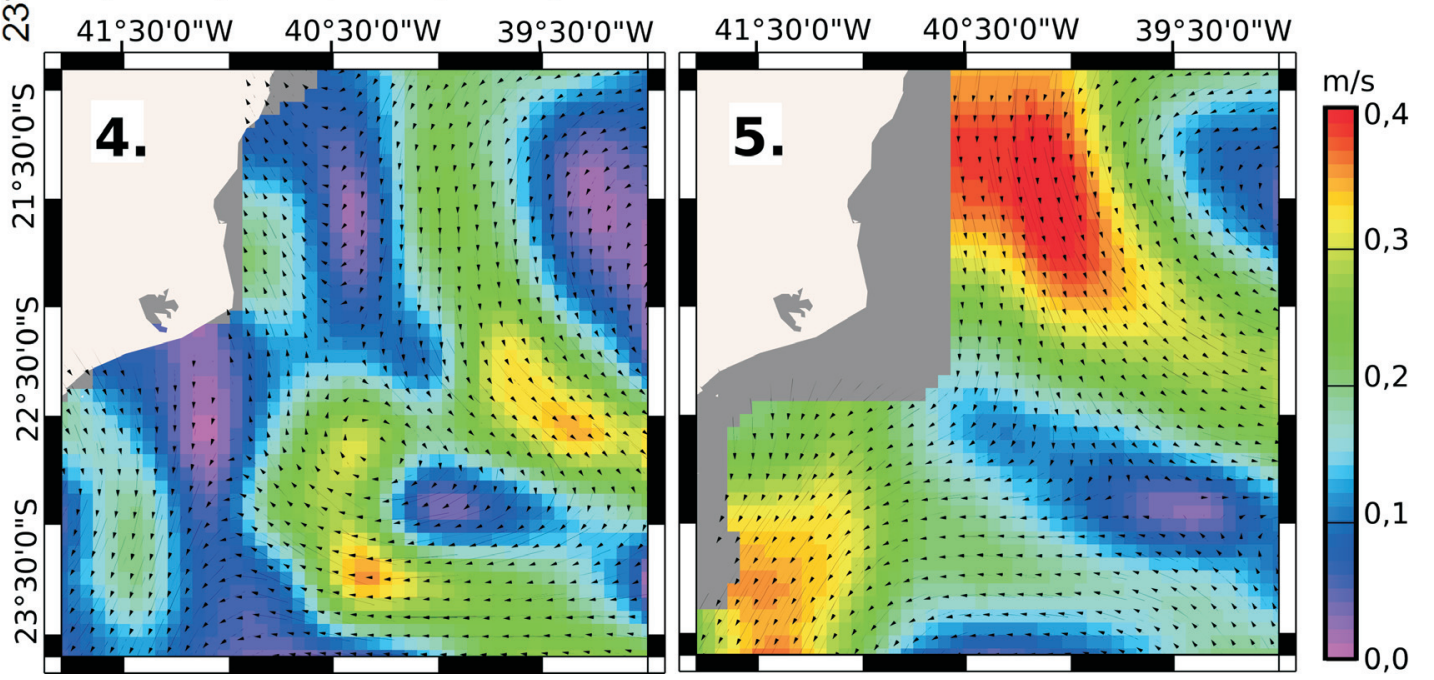

Figura 7 - Feições oceanográficas observadas em imagem MODIS Aqua em condição geométrica de sunglint de 2/1/2010 às 16h20min GMT. (1) Composição colorida (R: $645 \mathrm{~nm}, \mathrm{G} 555 \mathrm{~nm}$ e B: $469 \mathrm{~nm}$ ) obtida pela fusão com a banda do infravermelho próximo ( $859 \mathrm{~nm}$ ). (2) Infravermelho próximo com realce na área de intensa convergência (ver texto). (3) Imagem esquemática descrevendo as principais feições sobrepostas à TSM. As letras denotam os domínios de AT - Água Costeira (A), vórtice de São Tomé (B) e AC - Água Costeira (C). (4) Corrente na superfície do mar de 1/1/2010 da base de dados Ocean Surface Current Analyses Real-time (OSCAR) (Bonjean e Lagerloef, 2002) e (5) corrente geostrófica de 2/1/2010 estimada por altimetria, calculada por Salto/Ducas e disponibilizado pela site da AVISO. 
cial e consequentemente a rugosidade (Espedal et al., 1996). Estes filmes na superfície do oceano são classificados principalmente em duas classes: filmes biogênicos e filmes minerais.

Os filmes biogênicos são originados principalmente pelo metabolismo do fitoplâncton e emergem na superfície em forma de camadas monomoleculares (Ermakov et al., 1992). Consequentemente, podem cobrir extensas áreas e interferir significativamente nas interações entre atmosfera e oceano, como por exemplo, nas trocas de gases, calor, partículas e momentum (Lin et al., 2003). A composição dos filmes biogênicos é diversa, mas na maioria dos casos inclui proteínas, lipídios, ácidos orgânicos, sacarídeos e metais associados à matéria orgânica (Alpers e Espedal, 2004).

Filmes minerais ocorrem em geral no oceano devido a exsudações naturais, vazamentos ligados à exploração de petróleo ou simples descartes de embarcações. Portanto, a geometria das manchas formadas por filmes minerais está normalmente associada a um ponto de origem, seja ele uma embarcação, plataforma ou um ponto no leito oceânico. Geralmente, apresentam uma forma mais alongada associada à deriva das correntes oceânicas e ao vento. Algumas das características como composição, viscosidade e solubilidade podem ser avaliadas por sensores remotos hiperespectrais e multiespectrais como descrito por Lammoglia e Souza Fillho (2012) em experimento realizado na Bacia de Campos, RJ.

A aglomeração destas finas camadas $(\sim 1 \mathrm{~nm})$ sobre a superfície é controlada pela dinâmica do vento. Se a velocidade do vento exceder um determinado limiar, os surfactantes são dissipados na coluna d'água devido a processos turbulentos na camada de mistura (Mariano et al., 2011). A resistência aos processos turbulentos está relacionada a composição destes filmes. Ermakov et al. (1992) sugerem que filmes biogênicos se dissipam quando o vento na superfície do mar alcança o limite de 6-7 m/s. Por sua vez, de acordo com Espedal (1999) filmes que permanecem detectáveis em imagens SAR com ventos próximos de 7-8 m/s têm alta probabilidade de serem de origem mineral, ou seja, hidrocarbonetos.

A Fig. 8 mostra a presença de filmes biogênicos sobre a plataforma continental adjacente à costa do Rio de Janeiro, entre a Baía de Guanabara, Cabo Frio e Cabo de São Tomé. A imagem do sensor MODIS Terra foi adquirida em 5/1/2013, 13:15 GMT. A composição colorida (R: 645 nm, G: $555 \mathrm{~nm}$ e B: $459 \mathrm{~nm}$ ) foi gerada após o processo de fusão com a banda do infravermelho próximo $(859 \mathrm{~nm})$ de modo a realçar a presença dos surfactantes. Estes filmes estão organizados em forma de bandas finas e alongadas acompanhando a dinâmica oceanográfica local. Assim como descrito nos estudos de Calado et al. (2010), Lorenzzetti et al. (2009), Signorini (1978) e Soutelino et al. (2011), a dinâmica local possui singularidades devido à ressurgência costeira, processos da plataforma continental, plumas de rios, presença da Zona de Convergência do Atlântico Sul e à influência da Corrente do Brasil, corrente de borda oeste com substancial atividade de mesoescala e turbulência. Filmes detectados em imagens na condição de sunglint podem efetivamente auxiliar na compreensão sobre a dinâmica da costa brasileira de forma similar a utilização por Munk (2001) no estudo global sobre feições criadas por vórtices.

A $r_{g}$ foi modelada considerando-se para água limpa e surfactantes os parâmetros de aquisição da Imagem MODIS Terra de 5/1/2013, 13:15 GMT (Fig. 8), com $\theta_{\text {sol }}$ $27,5^{\circ}, \varphi 11,7^{\circ}$, direção do vento $145^{\circ}$ e magnitude do vento $4,2 \mathrm{~m} / \mathrm{s}$. O resultado desta modelagem (Fig. 9) demonstra a diferente resposta para áreas com surfactantes em relação à água limpa. À medida que a geometria de aquisição se aproxima da reflexão especular, ambas as curvas alcançam o máximo sunglint. Porém, a curva de surfactantes é mais intensa e alongada enquanto a modelagem para água limpa tem pico mais suavizado. Consequentemente, nestas dadas condições, os filmes biogênicos aparecem com contraste positivo em relação a águas limpas, ou seja, mais brilhantes em relação à cena.

Dependendo da geometria de aquisição, magnitude e direção do vento os surfactantes podem ser detectados em imagens na condição de sunglint com um contraste escuro, ou seja, menos brilhantes em relação à cena. Como exemplo deste processo, a Fig. 10 ilustra a detecção de filmes minerais com contraste negativo pelo sensor MODIS na condição de sunglint, na detecção do filme mineral do vazamento de óleo (hidrocarboneto) ocorrido em novembro de 2011, no campo de Frade, litoral norte do RJ.

$\mathrm{O}$ contraste negativo apresentado na imagem MODIS Aqua de 12/11/2011 10:20 GMT também pode ser modelado com a $r_{g}$ (Fig. 11). Os parâmetros da geometria de aquisição são: $\theta_{\text {sol }} 27,2^{\circ}, \varphi 175^{\circ}$, direção do vento $220^{\circ} \mathrm{e}$ magnitude do vento $2,2 \mathrm{~m} / \mathrm{s}$. Observando a porção da mancha de óleo na posição $22,62^{\circ} \mathrm{S}$ e $42,50^{\circ} \mathrm{W}$ observa-se que a $r_{g}$ atribuída aos surfactantes é mais intensa que a $r_{g}$ de água limpa, mesmo que estes valores sejam muito próximos e a intensidade do sunglint seja baixa. A detecção é efetiva mesmo em condições de vento baixo, o que pode ser limitante para a detecção SAR onde o limiar de vento para formação de ondas de Bragg corresponde a $2 \mathrm{~m} / \mathrm{s}$.

Comparando as modelagens de $r_{g}$ (Figs. 9 e 11) observa-se que as curvas da cena adquirida em 5/1/2013 são mais intensas que as da cena de 12/11/2011. Os diferentes contrastes observados são mais relacionados às condições geométricas de imageamento e campo de vento na superfície do mar que ao tipo de filmes (biogênicos ou minerais). Alguns autores como Pisano et al. (2015) utilizam as relações entre a modelagem do sunglint e radiâncias estimadas pelo MODIS para a deteção automática de filmes surfactantes.

A importância da modelagem do $r_{g}$ pode ser exemplificada ao analisar as características desta mancha de 


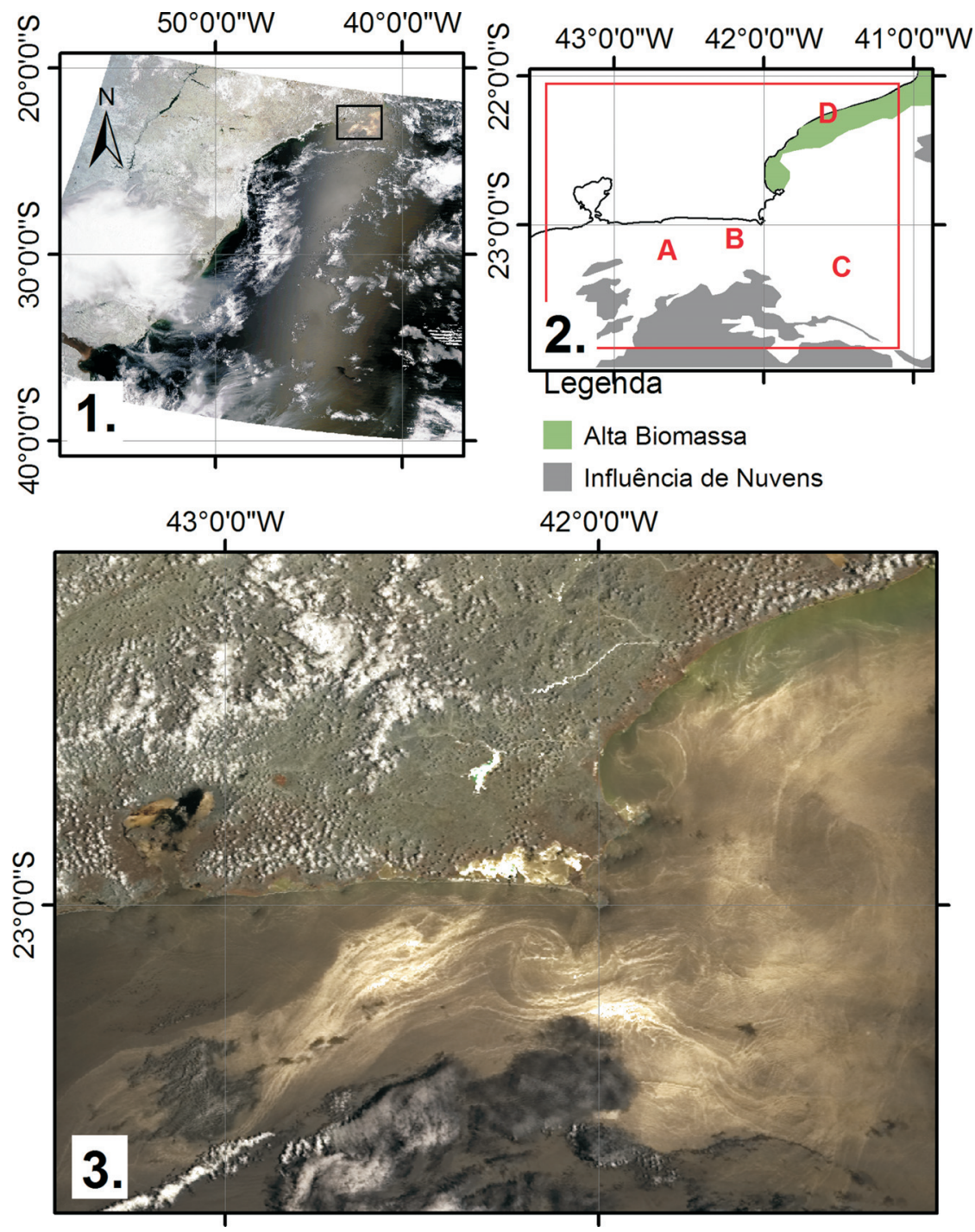

Figura 8 - Imagem MODIS Terra de 5/1/2013, 13:15 GMT, na condição geométrica de sunglint. A composição colorida (R: 645 nm, G: 555 nm e B: 469 nm) mostra a área marinha em coloração amarelada devido à contaminação por reflexão especular entre sol, superfície e água (1). Na área de destaque (3) o sunglint permite diferenciar filmes biogênicos na região da Bacia de Campos e arredores de Cabo Frio, RJ. O quadro esquemático (2) descreve a ocorrência de filmes biogênicos na área de destaque em 4 domínios: (A) advectados pela Corrente do Brasil, (B) sob influência de feições de mesoescala, (C) filmes em área de ondas internas solitárias e (D) em área de elevada biomassa fitoplanctônica.

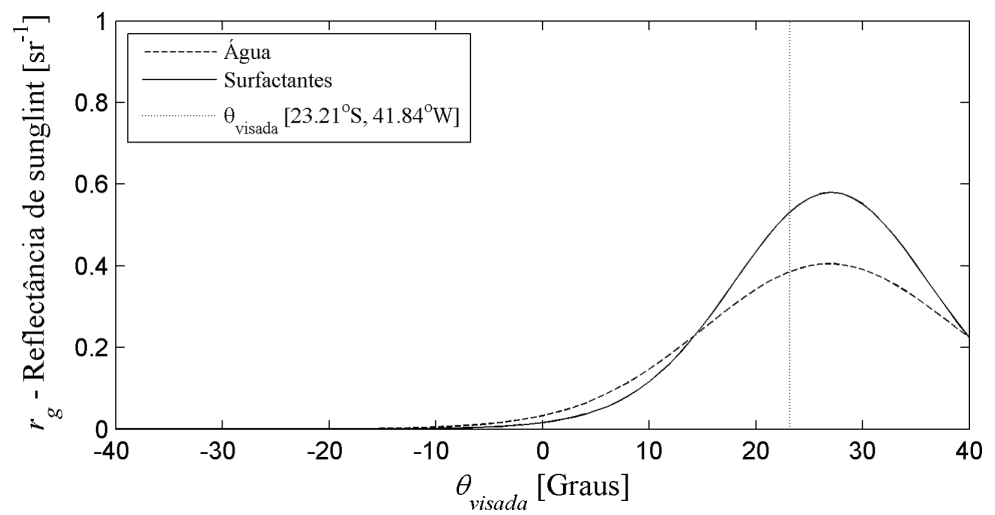

Figura 9 - Reflectancia de sunglint $\left(r_{g}\right)$ modelada para imagem MODIS Terra de 5/1/2013 13:15 GMT. As curvas de $r_{g}$ foram modeladas para água limpa (linha tracejada) e água com presenca de surfactantes (linha continua). Os picos das curvas são posicionados proximos a $\theta_{\text {visada }} 28^{\circ}$, resultando na detecção de filmes com um contraste positivo e brilho intenso. Os parametros da modelagem do $r_{g}$ considerados sao: $\theta_{\text {sol }} 27,5^{\circ}, \varphi 11,7^{\circ}$, direção do vento $145^{\circ} \mathrm{e}$ magnitude do vento $4,2 \mathrm{~m} / \mathrm{s}$, na posicao $23,21^{\circ} \mathrm{S}$ e $41,84^{\circ} \mathrm{W}$. 


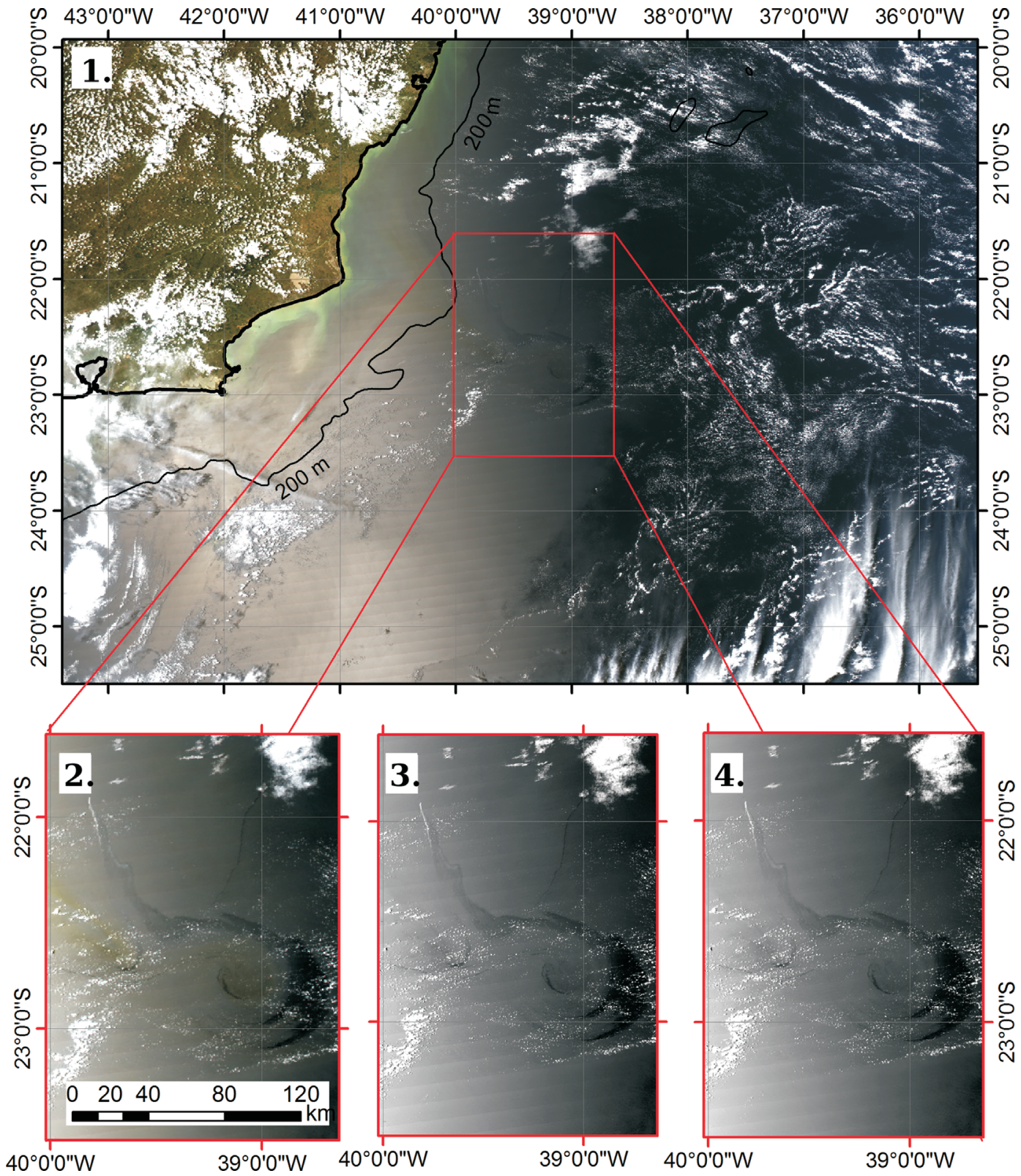

Figura 10 - (1) Imagem Aqua MODIS na condição de sunglint em 12/11/2011 10:20 GMT em composição colorida com resolução de 500 m. (2) Destaque da mancha produzida pelo vazamento de óleo no campo de Frade. (3) Destaque na resolução de 250 m na banda do infravermelho próximo (859 $\mathrm{nm})$ e (4) banda do vermelho $(645 \mathrm{~nm})$. Os filmes minerais são identificados por um contraste negativo em relação à cena, ou seja, mais escuro em relação aos demais pixels presentes no sunglint (ver texto para explicação).

óleo. No foco do vazamento de óleo, como já apresentada na Fig. 10, há um filamento com contraste positivo, isto é, brilhante em relação à cena. Conforme a mancha é advectada pela dinâmica local esse contraste torna-se negativo, ou seja, a mancha de óleo aparece mais escura. O contraste positivo no foco do vazamento contraria a modelagem do $r_{g}$, uma vez que é prevista uma mancha de óleo escura. Esta diferença entre o modelo e a imagem MODIS resulta da emulsão água-óleo no interior da coluna d'água concentrada próximo ao foco do vazamento. Esta feição brilhante observada nesta parte da imagem MODIS não é devido à reflexão de sunglint.
Imagens ópticas na condição de sunglint e imagens SAR em associação, podem melhorar a detecção de vazamentos de óleo (Leifer et al., 2012), garantindo assim uma melhor frequência de amostragem, o que é fundamental para efetiva caracterização e contenção dessas manchas. Principalmente, porque diversos agravantes são problemas frequentes, como variações na dinâmica local, correntes, condições de vento, turbulência na camada de mistura e mesmo os altos custos de operações marítimas. As limitações inerentes a cada sensor, como resolução espacial e tempo de revisita, também podem resultar em uma detecção apenas parcial da abrangência dos filmes. Como dis- 


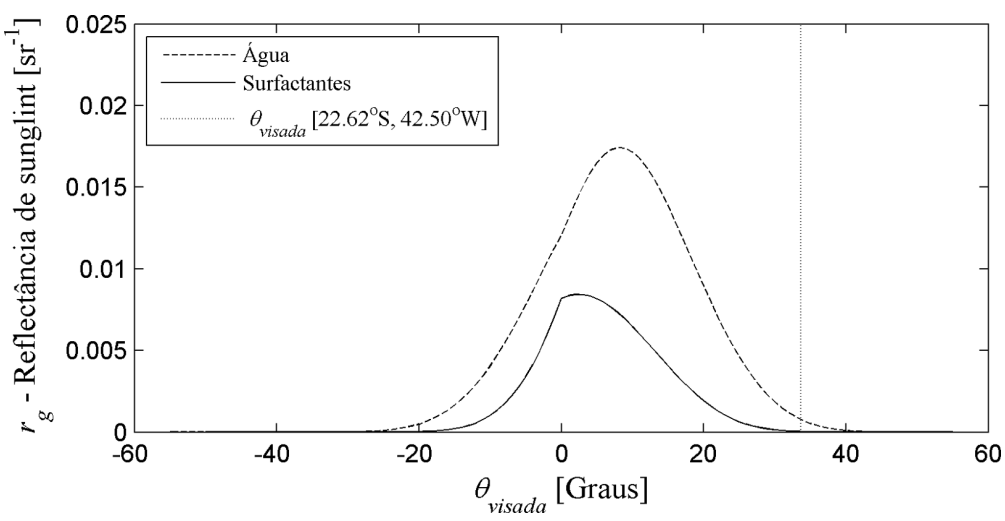

Figura 11 - Reflectância de sunglint $\left(r_{g}\right)$ modelada para imagem MODIS Aqua de 12/11/2011 10:20 GMT. As curvas de $r_{g}$ modeladas para água limpa (linha tracejada) e agua com presenca de surfactantes (linha continua) possui picos centrados nos angulo de reflexão especular entre o sol, superficie e sensor. A diferente posição dos picos, tanto em relação a $r_{g}$ máxima e em relação a visada do sensor, ocorre devido ao comportamento diferente da rugosidade para agua limpa e água com surfactantes. Parâmetros da modelagem do $r_{g}$ são: $\theta_{\text {sol }} 27,2^{\circ}, \varphi 175^{\circ}$.

cutido por Ivanov et al. (2002), filmes minerais detectados em imagens SAR representam, em média, menos que 10\% da área real das manchas geradas por um vazamento.

Como exemplo da sinergia de sensores, a imagem $\mathrm{Ad}$ vanced Synthetic Aperture Radar (ASAR) do satélite Envisat sobre o vazamento de óleo da plataforma Chevron, dia 25/11/2011, 12:15 GMT, mostra a permanência de filmes minerais na região da Bacia de Frade, RJ (Fig. 12). Estas manchas são demarcadas pelo baixo sigma-zero, devido à supressão de ondas capilares. Os filmes minerais detectados na imagem ASAR tem formato de bandas paralelas, o comprimento pode alcançar $174 \mathrm{~km}$ e larguras em torno de $250 \mathrm{~m}$. Alvos metálicos como embarcações e plataformas de exploração e produção de petróleo são demarcados por intenso sigma-zero, nas demais áreas prevalece o ruído speckle (padrão sal-pimenta) típico de imagens SAR. Ambas as observações, pelo sensor ASAR e sensor MODIS na condição de sunglint revelam que os filmes foram advectados pela dinâmica oceanográfica do vórtice de São Tomé (Calado et al., 2010), litoral norte do RJ.

\section{Ondas Internas Solitárias}

As ondas internas solitárias (OIS) são fenômenos de dissipação da energia de maré interna sobre a plataforma continental, se propagando pelas isopicnais em áreas estratificadas. A passagem de um trem de OIS altera os padrões de correntes próximas à superfície gerando bandas de convergências e divergências. Desta forma, o padrão de rugosidade marinha é alterado afetando o padrão do sunglint (Jackson, 2007). Este processo dinâmico é também frequentemente acompanhado de material oleaginoso quando associado a incrementos da produtividade marinha (Cox e Munk, 1956). Ambos os processos permitem que ondas internas solitárias de subsuperfície sejam detectadas em imagens na condição de sunglint (Pingree e New, 1995). O padrão espacial das ondas internas é caracterizado por linhas de ondas concêntricas como as observadas na imagens
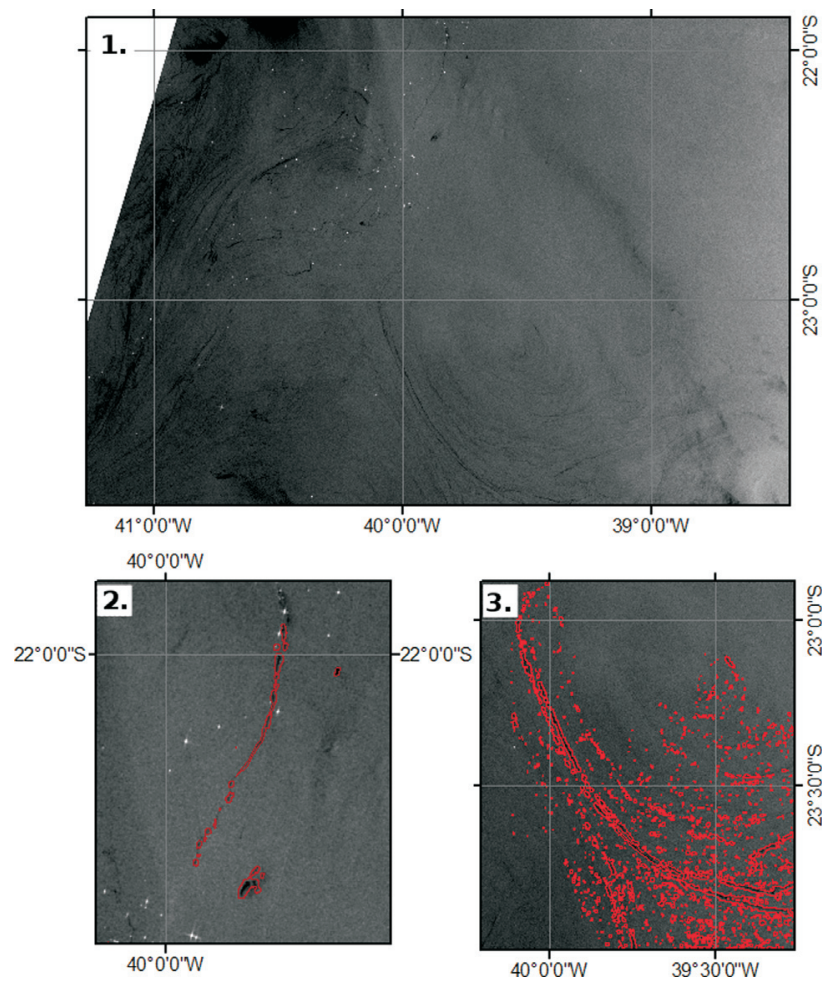

Figura 12 - Imagem Envista ASAR no modo Wide Swat Image de 25/11/2011 às 11:25 GMT (1), sobre a Bacia Sedmentar do Frade, RJ, próxima a Costa Sudeste Brasileira. As áreas selecionadas (2 e 3) foram extraídas da mesma imagem ASAR e demonstram o resultado da detecção de filmes minerais pelo método de classificação textural por semivariograma Unsupervised Semivariogram Textural Classifier (USTC), como descrito por Pellon et al. (2004). Detecção de óleo próximo à plataforma da Chevron (2) caracterizada pelo baixo retroespalhamento (alvo escuro) e demarcado pelas linhas vermelhas. Feições de filmes minerais capturados pelo vórtice oceanográfico de São Tomé (3).

MODIS na condição de sunglint da região de Cabo Frio, RJ (Fig. 13).

As OIS são originadas pela maré interna baroclínica, que ao interagir com abruptos gradientes da topografia de 


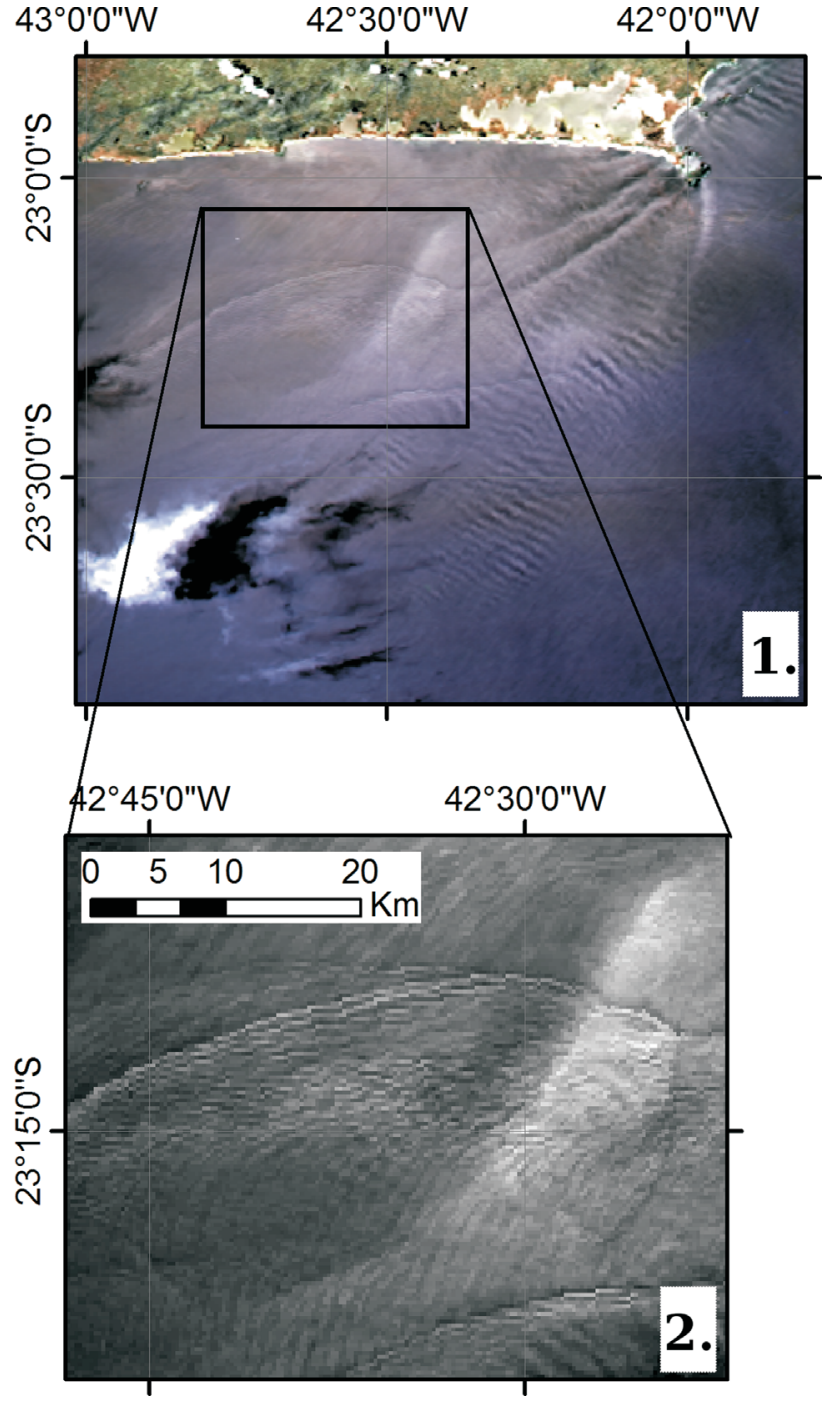

Figura 13 - Exemplo de ondas intenas solitárias (OIS) observadas sobre a plataforma continental próximo à Cabo Frio, RJ em imagem MODIS na condição geométrica de sunglint adquirida em 9/1/2010. (1) Composição colorida (R:645 nm, G: $555 \mathrm{~nm}$ e B: $469 \mathrm{~nm}$ ) e (2) OIS detectadas pela banda do infravermelho próximo $(859 \mathrm{~nm})$ em resolução espacial de $250 \mathrm{~m}$.

fundo, em condição de coluna d'água estratificada, provocam uma oscilação da termoclina que se propaga com o efeito da maré. A quebra da plataforma continental é considerada uma região mais propícia à formação das OIS devido à sua declividade. Como descrito por Pingree e New (1995) as OIS são formadas preferencialmente em pontos de uma abrupta variação da batimetria $\boldsymbol{c}$ (Eq. (9)):

$$
\boldsymbol{c}= \pm\left(\frac{\sigma_{M 2}^{2}-f^{2}}{N^{2}-\sigma_{M 2}^{2}}\right)
$$

considerando a estabilidade da termoclina pela frequência de Brunt-Vaisala $N^{2}$, o parâmetro de Coriolis $f$ e frequência do ciclo semi-diurno da maré lunar $\sigma^{2}{ }_{M 2}$.
Lorenzzetti e Dias (2013) fizeram um levantamento das OIS na costa sudeste brasileira a partir de imagens do sensor ASAR. A ocorrência das OIS foi relacionada à componente de maré semi-diurna, às variações sazonais de estabilidade da coluna d'água e confinadas ao regime de vento na superfície do mar propício para a formação das ondas Bragg. De acordo com esses autores, a força da ação de maré baroclínica exercida sobre a topografia de fundo $F$, pode ser quantificada de acordo com a Eq. (10):

$$
F=\frac{1}{\omega_{m}} Q \nabla\left(\frac{1}{h}\right) \int_{H}^{0} z N^{2}(z) d z
$$

onde $\omega_{m}$ é a frequência angular da maré $\left(\mathrm{rad} \mathrm{s}^{-1}\right), Z$ é a profundidade, com a superfície $Z=0, Q$ é o vetor de fluxo barotrópico da maré $Q=\left(Q_{x}, Q_{y}\right)=\left(u_{l} H, v_{l} H\right)$, sendo $u_{l}$ e $v_{I}$ seus componentes zonais e meridionais, $H$ é a posição na coluna d'água e $h(x, y)$ a profundidade.

Algumas propriedades oceanográficas podem ser estimadas a partir da análise da geometria das manifestações das OIS em imagens de satélite, como por exemplo, a profundidade da camada de mistura (Li et al., 2000), frequência de Brunt-Vaissala (Zheng et al., 1993) e calor armazenado (Mollo-Christensen e Mascarenhas, 1979).

Jackson (2007) fez um levantamento global dos locais de geração das OIS, utilizando apenas o sensor MODIS em condição de sunglint, no período entre agosto de 2002 e maio de 2004. De acordo com este autor, a cobertura global diária do sensor MODIS permitiu observar o mesmo pacote de OIS em aquisições quase simultâneas por outros sensores SAR ou ópticos na condição de sunglint.

A capacidade de detectar as OIS através de sensores ópticos na condição de sunglint depende de uma geometria de aquisição específica e da resolução espacial inerente a cada sensor. A amostragem espacial adequada para o estudo de ondas é determinada pela frequência crítica de Nyquist, $N_{y}=1 / 2 \delta$, ou seja, deve ser no mínimo metade do comprimento de onda. Lorenzzetti e Dias (2013) estimaram os comprimentos máximos e mínimos das OIS de 2,36 km e $0,22 \mathrm{~km}$, respectivamente. Sendo assim, as imagens adquiridas com resolução de $250 \mathrm{~m}$ tem uma capacidade limitada de observação dos comprimentos de onda menores que $500 \mathrm{~m}$. Dados com resolução de $500 \mathrm{~m}$ e $1 \mathrm{~km}$ possuem uma limitação ainda maior considerando a frequência de Nyquist. Estas limitações são ainda mais relevantes uma vez que os pacotes de OIS têm comprimentos de onda médio iguais a $1,04 \mathrm{~km}, 0,9 \mathrm{~km}$ e $0,8 \mathrm{~km}$, respectivamente para o $1^{\circ}, 2^{\circ}$ e $3^{\circ}$ pacotes de OIS.

Ondas internas, da mesma forma que as ondas de superfície, podem apresentar o fenômeno de arrebentação. Isto pode promover a ressuspensão de sedimentos de fundo na plataforma continental, trazendo nutrientes para a camada eufótica. Segundo Longhurst e Pauly (1987), os processos de agitação mecânica e turbulência causados por arrebentação de ondas internas afetam o fitoplâncton, podendo 
catalisar o acréscimo na produtividade marinha. $\mathrm{O}$ acréscimo na biomassa fitoplanctônica devido às OIS foi avaliado por Muacho et al. (2014) na baía de Biscay, ao sul da Península Ibérica. Segundo Pan et al. (2012), o acréscimo de biomassa observado no Mar do Sul da China, resulta do aumento de produção primária em resposta à entrada de nutrientes na camada eufótica provocada pela oscilação da termoclina devido à ação das OIS.

\section{Influência da Atmosfera: Vento, Rolls e Outros Efeitos}

Fenômenos atmosféricos e o atrito pelo vento alteram os padrões de rugosidade da superfície do mar devido à formação de ondas capilares ou modulações no espectro das ondas capilares. Vários autores como O’Brien e Mitchell (1988), Wald e Monget (1983), Wylie et al. (1981), e Cox e Munk (1954) já haviam utilizado imagens na condição de sunglint para o cálculo da velocidade do vento em superfície. Mais recentemente, Harmel e Chami (2012) demonstraram que o uso de sensores ópticos passivos, multidirecionais e polarimétricos, nas bandas do visível e infravermelho próximo provém uma alternativa para as medidas de vento na superfície do mar. Os resultados alcançando foram satisfatórios quando comparados a medidas de vento in-situ (rms > $0,84)$, com resolução espacial mais refinada (6 km no nadir) em relação a estimativas típicas de vento por escaterômetro ou radiometria passiva de micro-ondas.

Em áreas costeiras, formações de relevo nas proximidades da costa ou em ilhas podem interferir nos campos de

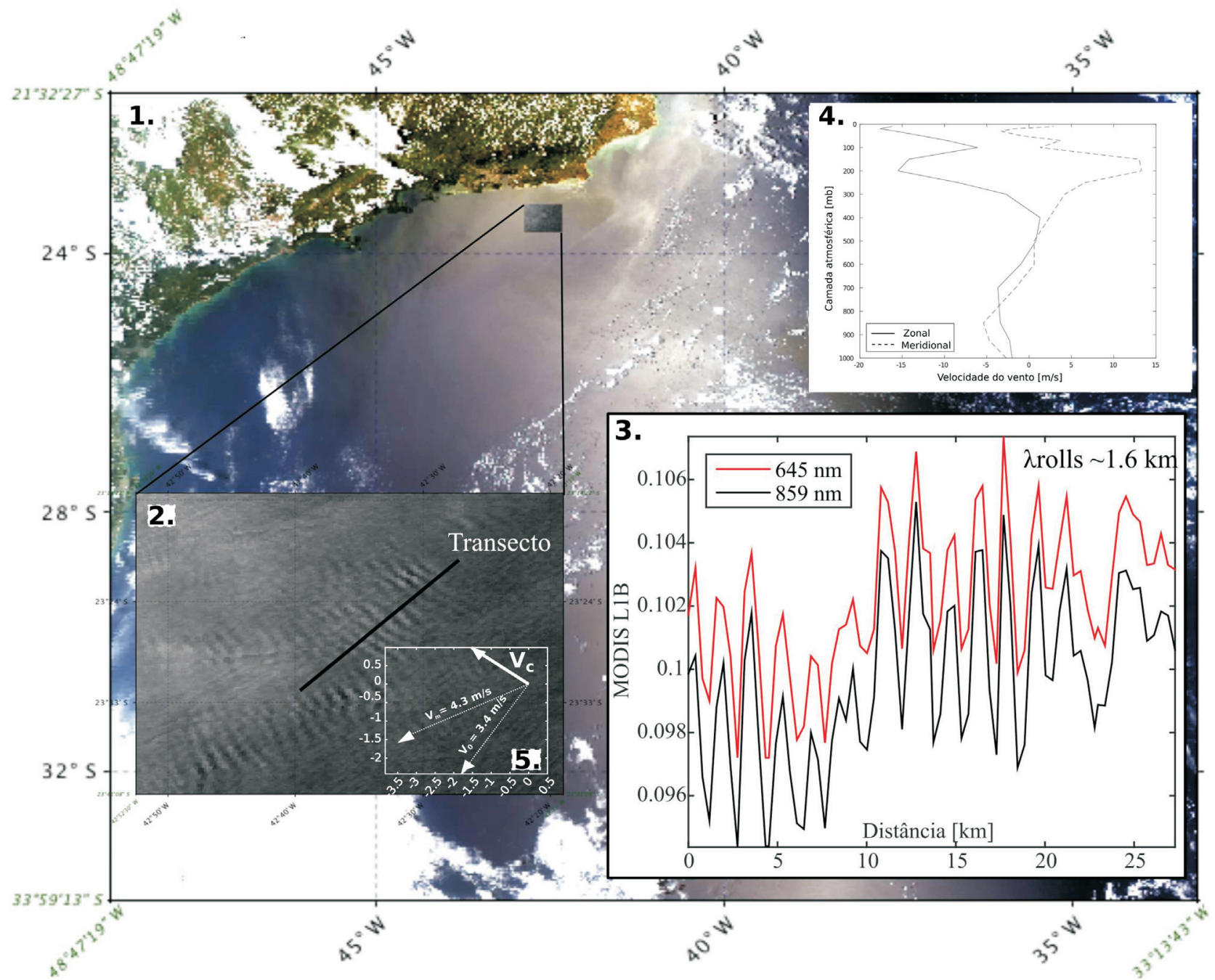

Figura 14 - Imagem MODIS Terra na condição de sunglint sobre o litoral sudeste do Brasil, adquirida em 12/2/2015 às 13:15 GMT. (1) Composição colorida (R:645 nm, G: $555 \mathrm{~nm}$ e B: $469 \mathrm{~nm}$ ). (2) A região ampliada mostra rolos (rolls) atmosféricos discriminados na banda do infravermelho próximo $(859 \mathrm{~nm})$ com resolução espacial de $250 \mathrm{~m}$. (3) O transecto extraido das bandas do vermelho (linha vermelha) e infravermelho próximo (linha preta) permitem inferir informações sobre a extrutura destes rolls, como por exemplo, o comprimento de onda $\lambda \cong 1.6 \mathrm{~km}$. (4) O perfil atmosférico da velocidade do vento zonal e meridional extraído da Reanálise NCEP/NCAR demonstra a variação do vento entre a camada de superfície e o topo da camada limite atmosférica. (5) Os rolls atmosféricos estão alinhados com o vetor de cizalhamento do vento $\mathrm{V}_{\mathrm{c}}$ calculado pelo vento na superfície $V_{0}$ e o vento na porção superior da camada limite $V_{m}$. 
vento (Chelton et al., 2004), criando padrões de divergência e convergência. Este efeito orográfico produz alterações na rugosidade a sotavento, resultando em feições observáveis nas imagens com sunglint. Já em imagens SAR, o efeito do relevo sobre o campo de ventos costeiros resulta em baixo retroespalhamento ou efeito de sombra (Clemente-Colón e Yan, 2000). O efeito orográfico pode também gerar ondas de gravidade semi-estacionárias na camada limite oceano-atmosfera, com comprimentos entre 6-7 km denominadas ondas de sotavento (Wurtele e Shaman, 1996). O efeito na rugosidade da superfície pode alterar o padrão de brilho nas imagens em condição de sunglint ou o retroespalhamento SAR, de acordo com a posição das cristas destas ondas de sotavento (Vachon et al., 1994).

Processos na camada limite oceano-atmosfera, como instabilidade dinâmica termal forçada pela diferença entre a direção do vento em superfície e vento geostrófico, podem gerar feições denominadas rolls atmosféricos. Segundo uma caracterização feita por Etling e Brown (1993) rolls são estruturas bem organizadas de vórtices com extensão alongada no sentido horizontal da borda planetária com comprimento entre 10 e $1000 \mathrm{~km}$, com cristas paralelas de comprimentos de ondas entre 2-10 km. De acordo com Sikora e Ufferman (2004), o eixo horizontal dos rolls atmosféricos são orientados pelo vetor de cisalhamento do vento na camada limite planetária $V_{c}$. A Fig. 14 mostra um exemplo de ocorrência de rolls atmosféricos na região da Bacia de Campos, RJ, observada pelo sensor MODIS Terra na condição de sunglint em 12/02/2015 às 13:15 GMT. Estimamos o $V_{c}$ com base no perfil atmosférico da reanálise NCEP/NCAR. Conforme explícito na Fig. 14, os eixos horizontais dos rolls coincidem com vetor cisalhamento $V_{c}$, estimado entre a superfície do mar e a camada de $700 \mathrm{Mb}$, localizada na parte superior da camada limite planetária.

Os rolls atmosféricos contribuem para os fluxos verticais de momentum, calor e umidade e influenciam também o desenvolvimento de convecção profunda (Young et al., 2002). A dinâmica destas feições coerentes pode resultar em modulações na rugosidade, devido aos movimentos das parcelas de ar acompanhando a circulação destes rolls e também devido aos movimentos verticais ascendentes (descendentes) das parcelas de ar gerando respectivamente bandas de alisamento (rugosidade) na superfície marinha. Essa modulação na rugosidade da superfície possibilita identificar a presença destas feições em imagens SAR (Alpers e Brümmer 1994; Müller et al., 2013), assim como, em imagens ópticas em condição de sunglint (Kapustin et al., 2012).

Um exemplo da complexidade de imagens na condição de sunglint em função de diferentes processos ambientais pode ser observado na Fig. 15. Nestas duas bandas do sensor MODIS Terra (centradas em $645 \mathrm{~nm}$ e $859 \mathrm{~nm}$ ) adquiridas em 6/1/2013 observam-se feições formadas por rolls atmosféricos, além de OIS e sombras de nuvens.
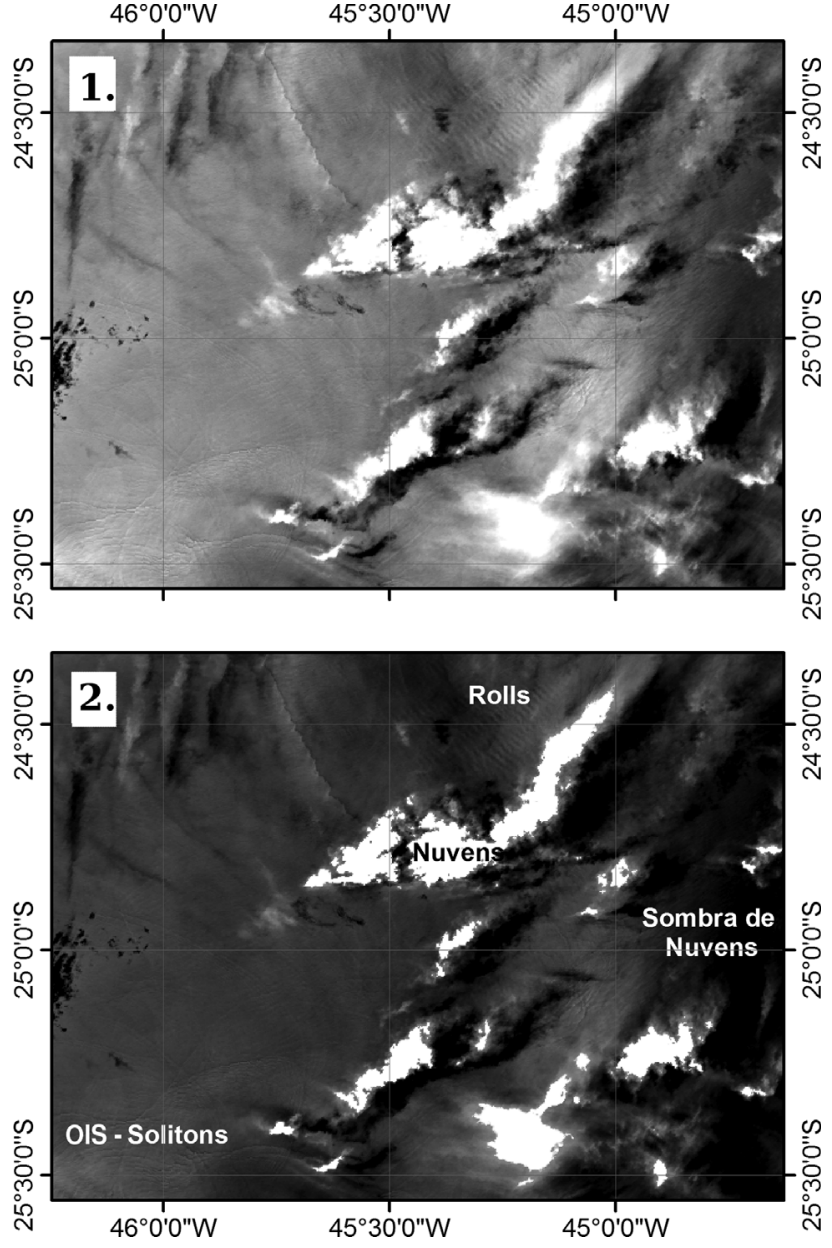

Figura 15 - Imagem MODIS Aqua em condição geométrica de sunglint adquirida em 6/1/2013 com resolução espacial de $250 \mathrm{~m}$. (1) Banda 645 $\mathrm{nm}$ (vermelho) e (2) banda $859 \mathrm{~nm}$ (infravermelho próximo). São distinguidas as seguintes feições impressas na assinatura do sunglint: rolls atmosféricos, OIS, além da ocorrência de nuvens e suas respectivas sombras.

\section{Conclusões e Considerações Finais}

Apresentamos neste trabalho exemplos de imagens ópticas em condição de sunglint utilizadas para a deteç̧ão de feições oceânicas e atmosféricas. Procuramos descrever os mecanismos físicos que possibilitam a identificação de feições nas imagens. Esperamos ter demonstrado a aplicabilidade das imagens com sunglint para estudos oceanográficos na costa brasileira. É claro que o uso dessa técnica não é possível em condição de cobertura de nuvens, à noite, ou durante alguns períodos do ano em que a condição de sunglint não é obtida. Porém, a grande disponibilidade de imagens orbitais gratuitas e de fácil acesso estimulam a sua utilização como sugerido aqui. Para a região da costa SE do Brasil, uma análise quantitativa das condições geométricas de aquisição para sensores de amplo campo de visada como o MODIS, indica que os períodos entre janeiro-fevereiro e 
outubro-dezembro são os mais adequados para a obtenção de imagens na condição de sunglint.

As imagens apresentadas foram analisadas buscando-se uma conectividade entre as feições observadas e a dinâmica local, de modo a subsidiar as interpretações e monitoramento do comportamento da Corrente do Brasil, bem como de seus vórtices e meandros. Trabalhos futuros dedicados a estudos sobre os processos oceanográficos e atmosféricos associados a modulações da rugosidade da superfície do mar podem ser beneficiados com a utilização de imagens na condição de sunglint, aumentando consideravelmente a amostragem espaço-temporal. Quando ocorre, o efeito do sunglint é predominante sobre o sinal detectado por um sensor orbital no visível. Uma vez que existem diversos métodos para remoção do efeito de sunglint, torna-se possível extrair de uma mesma imagem os parâmetros de rugosidade e bio-ópticos da coluna d'água. Esta abordagem permitiria, por exemplo, avaliar simultaneamente os efeitos dos processos das ondas internas, como por exemplo, ressuspensão de sedimentos, ou incremento da produção primária

As áreas costeiras e oceânicas são suscetíveis à contaminação por vazamentos de hidrocarbonetos, principalmente aquelas localizadas próximas às áreas portuárias ou de exploração de petróleo e gás. Essas ocorrências podem acarretar graves danos ambientais. A capacidade de identificar e monitorar esses vazamentos é de grande valia para auxiliar às ações de mitigação e redução dos impactos. A disponibilidade de imagens com sunglint pode adicionar informação importante para controlar e detectar vazamentos de óleo na superfície do mar em sinergia com outros métodos e sensores remotos, como o SAR, resultando em uma melhoria das ações de resposta e contenção e minimizando os prejuízos ambientais.

\section{Agradecimentos}

Agradecemos ao INPE/FUNCATE/PETROBRAS pelo financiamento segundo o Termo de Cooperação 0050.0066309.11.9.

\section{Referências}

ALPERS, W.; BRANDT, P.; LAZAR, A.; DAGORNE, D.; SOW, B.; FAYE, S.; HANSEN, M.W.; RUBINO, A.; POULAIN, P.M.; BREHMER, P. A small-scale oceanic eddy off the coast of West Africa studied by multi-sensor satellite and surface drifter data. Remote Sens Environ, v. 129, p. 132143, 2013.

ALPERS, W.; BRÜMMER, B. Atmospheric boundary layer rolls observed by the synthetic aperture radar aboard the ERS-1 satellite. J Geophys Res, v. 99, n. C6, p.12613-12621, 1994.

ALPERS, W.; ESPEDAL, H.A. Oils and surfactants. In: JACKSON, C. R.; APEL, J. R. (Eds.). Synthetic aperture radar marine user's manual. Washington: National Ocean and Atmospheric Administration, 2004. p. 263-275.

SIKORA, T.D.; UFFERMAN, S. Marine Boundary Layer Cellular Convection and Longitudinal Roll Vortices. In:
JACKSON, C.R.; APEL, J.R. (Eds.). Synthetic Aperture Radar Marine User's Manual. Washington: National Ocean and Atmospheric Administration, 2004, p. 321-330.

APEL, J.R.; GONZALEZ, F.I. Nonlinear features of internal waves off Baja California as observed from the SEASAT imaging radar. J Geophys Res, v. 88, n. C7, p. 4459-4466, 1983.

BAKUN, A. Fronts and eddies as key structures in the habitat of marine fish larvae?: opportunity, adaptive response and competitive advantage. Sci Mar, v.70, n. S2, p. 105-122, 2006.

BELKIN, I.M.; MILLER, P. Composite front maps for improved visibility of dynamic sea-surface features on cloudy SeaWiFS and AVHRR data. J Marine Syst, v. 78, n. 3, p. 327-336, 2009.

BELKIN, I.M.; O'REILLY, J.E. An algorithm for oceanic front detection in chlorophyll and SST satellite imagery. J Marine Syst, v. 78, n. 3, p. 319-326, 2009.

BENTZ, C.M.; LORENZZETTI, J.A.; KAMPEL, M. Multi-sensor synergistic analysis of mesoscale oceanic features: Campos Basin, south-eastern Brazil. Int J Remote Sens, v. 25, n. 21, p. 4835-4841, 2004.

BONJEAN, F.; LAGERLOEF, G.S.E. Diagnostic model and analysis of the surface currents in the tropical Pacific Ocean. J Phys Oceanogr, v. 32, n. 10, p. 2938-2954, 2002.

BRANDINI, F.P. Hydrography and characteristics of the phytoplankton in shelf and oceanic waters off southeastern Brazil during winter (July/August 1982) and summer (February/March 1984). Hydrobiologia,v. 196, n. 2, p. 111-148 (1990).

BRÉON, F.M.; HENRIOT, N. Spaceborne observations of ocean glint reflectance and modeling of wave slope distributions. J Geophys Res, v. 111, n. C6, p. C06005, 2006.

CALADO, L.; DA SILVEIRA, I.C.A.; GANGOPADHYAY, A.; DE CASTRO, B.M. Eddy-induced upwelling off Cape São Tomé (22 S, Brazil). Cont Shelf Res, v. 30, n. 10-11, p. 1181-1188, 2010.

CAMPOS, E.J.D., VELHOTE, D.; DA SILVEIRA, I.C.A. Shelf break upwelling driven by Brazil Current cyclonic meanders.Geophys Res Lett, v.27, n. 6, p. 751-754, 2000.

CASTELAO, R.M.; BARTH, J.A. Upwelling around Cabo Frio, Brazil: the importance of wind stress curl. Geophys Res Lett, v. 33, n. 3, p. L03602, 2006.

CHELTON, D.B.; SCHLAX, M.G.; FREILICH, M.H.; MILLIFF, R.F. Satellite measurements reveal persistent smallscale features in ocean winds. Science, v. 303, n. 5660, p. 978-83, 13 Feb. 2004.

CLEMENTE-COLON, P. Observations of east coast upwelling conditions in synthetic aperture radar imagery. IEEE Trans Geosci Remote Sens, v. 37, n. 5, p. 2239-2248, 1999.

CLEMENTE-COLÓN, P.; YAN, X. Low-backscatter ocean features in Synthetic Aperture Radar imagery. John Hopkins APL Tech Dig, v. 21, n. 1, p.116-121, 2000.

COX, C.; MUNK, W. Measurement of the roughness of the sea surface from photographs of the sun's glitter. J Opt Soc Am, v. 44, n. 11, p. 838-850, 1954.

COX, C.; MUNK, W. Slopes of the sea surface deduced from photographs of sun glitter. Berkeley: University of California, 1956. (Bulletin of the Scripts Institution of Oceanography) 
ERMAKOV, S.A.; SALASHIN, S.G.; PANCHENKO, A.R. Film slicks on the sea surface and some mechanisms of their formation. Dynam Atmos Oceans, v. 16, n. 3-4, p. 279-304, 1992.

ESPEDAL, H. Detection of oil spill and natural film in the marine environment by spaceborne SAR. In: GEOSCIENCE AND REMOTE SENSING SYMPOSIUM, 1999. Proceedings... IEEE, 1999. p.1478-1480.

ESPEDAL, H.A.; JOHANNESSEN, O.M.; KNULST, J.C. Satellite detection of natural films on the ocean surface. Geophys Res Lett, v. 23, p. 3151-3154, 1996.

ETLING, D.; BROWN, R.A. Roll vortices in the planetary boundary layer: a review. Bound-Lay Meteorol, v. 65, p. 215248, 1993.

FRULLA, L.A.; MILOVICH, J.A.; GAGLIARDINI, D.A. Illumination and observation geometry for NOAA-AVHRR images. Int J Remote Sens, v.16, n.12, p.2233-2253, 1995.

GADE, M.; ALPERS, W.; HÜHNERFUSS, H.; MASUKO, H.; KOBAYASHI, T. Imaging of biogenic and anthropogenic ocean surface films by the multifrequency/multipolarization SIR-C/X-SAR. J Geophys Res, v. 103, n. C9, p. 18851, 1998.

GAGLIARDINI, D.A.; COLÓN, P.C. Ocean feature Detection using microwave backscatter and sun glint observations. Gayana, v. 68, n. 2, p. 180-185, 2004.

GOWER, J.; HU, C.; BORSTAD, G.; KING, S. Ocean color satellites show extensive lines of floating sargassum in the Gulf of Mexico. IEEE Trans Geosci Remote Sens, v. 44, n. 12, p. 3619-3625, 2006.

HARMEL, T.; CHAMI, M. Determination of sea surface wind speed using the polarimetric and multidirectional properties of satellite measurements in visible bands. Geophys Res Lett, v. 39, n. 19, L19611, 2012.

HU, C.; LI, X.; PICHEL, W.G.; MULLER-KARGER, F.E. Detection of natural oil slicks in the NW Gulf of Mexico using MODIS imagery. Geophys Res Lett, v. 36, L01604, 2009.

$\mathrm{HU}, \mathrm{C}$. An empirical approach to derive MODIS ocean color patterns under severe sun glint. Geophys Res Lett, v. 38, L01603, 2011.

IVANOV, A.; HE, M.; FANG, M. Oil spill detection with the RADARSAT SAR in the waters of the Yellow and East China Sea: a case study. In: ASIAN CONFERENCE ON REMOTE SENSING, 23., 2002, Kathmandu, Nepal. Proceedings 2002. Disponível em http://www.gisdevelopment.net/aars/acrs/2002/sar/011.pdf.

JACKSON, C. Internal wave detection using the Moderate Resolution Imaging Spectroradiometer (MODIS). J Geophys Res, v. 112, n. C11, C11012, 2007.

JACKSON, C.R.; ALPERS, W. The role of the critical angle in brightness reversals on sunglint images of the sea surface. $\mathbf{J}$ Geophys Res, v. 115, n. C9, C09019, 2010.

JOHANNESSEN, J.A.; SHUCHMAN, R.A.; DIGRANES, G.; LYZENGA, D.R.; WACKERMAN, C.; JOHANNESSEN, O.M.; VACHON, P.W. Coastal ocean fronts and eddies imaged with ERS 1 synthetic aperture radar. J Geophys Res, v. 101, n. C3, p. 6651-6667, 1996.

KAPUSTIN, V.N.; CLARKE, A.D.; HOWELL, S.G.; MCNAUGHTON, C.S.; BREKHOVSKIKH, V.L.; ZHOU, J. Evaluating primary marine aerosol production and atmospheric roll structures in Hawaii's natural oceanic wind tunnel. J Atmos Ocean Tech, v. 29, n. 5, p. 668-682, 2012.
KARATHANASSI, V.; KOLOKOUSIS, P.; IOANNIDOU, S. A comparison study on fusion methods using evaluation indicators. Int J Remote Sens, v. 28, n.10, p.2309-2341, 2007.

KAY, S.; HEDLEY, J.D.; LAVENDER, S. Sun glint correction of high and low spatial resolution images of aquatic scenes: a review of methods for visible and near-infrared wavelengths. Remote Sens, v. 1, n. 4, p. 697-730, 2009.

KEHOE, K.E. Ocean sun glint albedo estimation from geostationay satellite data. Dissertação (Mestrado em Ciências Atmosféricas) - University of Arizona, 2002.

KHATTAK, S.; VAUGHAN, R.A.; CRACKNELL, A.P. Sunglint and its observation in AVHRR data. Remote Sens Environ, v. 37, n. 2, p. 101-116, 1991.

KUDRYAVTSEV, V.; MYASOEDOV, A.; CHAPRON, B.; JOHANNESSEN, J.A.; COLLARD, F. Imaging mesoscale upper ocean dynamics using synthetic aperture radar and optical data. J Geophys Res, v. 117, n. C4, p. C04029, 2012.

LAMMOGLIA, T.; SOUZA FILHO, C.R. Mapping and characterization of the API gravity of offshore hydrocarbon seepages using multispectral ASTER data. Remote Sens Environ, v. 123, p. 381-389, 2012.

LEIFER, I.; LEHR, W.J.; SIMECEK-BEATTY, D.; BRADLEY, E.; CLARK, R.; DENNISON, P.; HU, Y.; MATHESON, S.; JONES, C.E.; HOLT, B.; REIF, M. State of the art satellite and airborne marine oil spill remote sensing: application to the BP deepwater horizon oil spill. Remote Sens Environ, v. 124, p.185-209, 2012.

LI, X.; CLEMENTE-COLÓN, P.; FRIEDMAN, K.S. Estimating oceanic mixed-layer depth from internal wave evolution observed from Radarsat-1 SAR. John Hopkins APL Tech Dig, v. 21, n. 1, p. 130-135, 2000.

LIN I. I.; ALPERS, W.; LIU, W.T. First evidence for the detection of natural surface films by the QuikSCAT scatterometer. Geophys Res Lett, v. 30, p. 2-5, 2003.

LONGHURST, A.R.; PAULY, D. Ecologia dos oceanos tropicais. São Paulo: Edusp, 2007.

LORENZZETTI, J.A.; STECH, J.L.; MELLO FILHO, W.L.; ASSIREU, A.T. Satellite observation of Brazil current inshore thermal front in the SW South Atlantic: space/time variability and sea surface temperatures. Cont Shelf Res, v. 29, n. 17, p. 2061-2068, 2009.

LORENZZETTI, J.A.; DIAS, F.G. Internal solitary waves in the Brazilian SE continental shelf: observations by Synthetic Aperture Radar. Int J Oceanogr, v. 2013, p. 1-11, 2013.

MARIANO, A.J.; KOURAFALOU, V.H.; SRINIVASAN, A.; KANG, H.; HALLIWELL, G.R.; RYAN, E.H.; ROFFER, M. On the modeling of the 2010 Gulf of Mexico oil spill. Dynam Atmos Oceans, v. 52, n.1/2, p. 322-340, 2011.

MATTHEWS, J.P.; AWAJI, T. Synoptic mapping of internalwave motions and surface currents near the lombok strait using the along-track stereo sun glitter technique. Remote Sens Environ, v. 114, n. 8, p. 1765-1776, 2010.

MCCLAIN, C.R.; ARRIGO, K.R.; ESAIAS, W.; DARZI, M.; PATT, F.S.; EVANS, R.H.; BROWN, C.W.; BROWN, J.W.; BARNES, R.A.; KUMAR, L. SeaWiFS quality control masks and flags: initial algorithms and implementation strategy. In: McCLAIN, C. R. et al. (Eds.). SeaWiFS Algorithms. Greenbelt: Goddard Space Flight Center, 1995. p.3-7. (Sea WiFS Technical Report Series, 28 pt 1). 
MITNIK, L.M.; LOBANOV, V.B. Investigation of OyashioKuroshio frontal zone using alos PALSAR images and ancillary information. In: Proceedings IEEE INTERNATIONAL GEOSCIENCE AND REMOTE SENSING SYMPOSIUM, 2011. Disponível em: http://ieeexplore.ieee.org/lpdocs/epic03/wrapper.htm?arnumber $=6049450$. Acesso em: 11 jun. 2015 .

MOLLO-CHRISTENSEN, E.; MASCARENHAS, A. Heat storage in the oceanic upper mixed layer inferred from landsat data. Science, v. 203, n. 4381, p. 653-654, 1979.

MONALDO, F.M.; THOMPSON, D.R.; BEAL, R.C.; PICHEL, W.G.; CLEMENTE-COLÓN, P. Comparison of SARderived wind speed with model predictions and ocean buoy measurements. IEEE Trans Geosci Remote Sens, v. 39, n. 12, p. 2587-2600, 2001.

MONZON, C.; FORESTER, D.W.; BURKHART, R.; BELLEMARE, J. Rough ocean surface and sunglint region characteristics. Appl Optics, v. 45, n. 27, p. 7089-7096, 2006.

MUACHO, S.; DA SILVA, J.C.B.; BROTAS, V.; OLIVEIRA, P.B. Effect of internal waves on near-surface chlorophyll concentration and primary production in the Nazaré Canyon (west of the Iberian Peninsula). Deep-Sea Res Pt I, v. 81, p. 89-96, 2013.

MUACHO, S.; DA SILVA, J.C.B.; BROTAS, V.; OLIVEIRA, P.B.; MAGALHAES, J.M. Chlorophyll enhancement in the central region of the Bay of Biscay as a result of internal tidal wave interaction. J Marine Syst, v. 136, n. 1, p. 22-30, 2014.

MÜLLER, S.; STANEV, E.V.; SCHULZ-STELLENFLETH, J.; STANEVA, J.; KOCH, W. Atmospheric boundary layer rolls: quantification of their effect on the hydrodynamics in the german bight. J Geophys Res - Oceans, v. 118, n. 10, p. $5036-5053,2013$.

MUNK, W. Spirals on the sea. Sci Mar, v. 65, n. S2, p. 193-198, 2001.

O'BRIEN, D.M.; MITCHELL, R.M. Retrieval of surface wind speed and aerosol optical depth over the oceans from AVHRR images of sun glint. J Appl Meteorol, v. 27, n. 12, p. $1395-1403,1988$.

OTTAVIANI, M.; SPURR, R.; STAMNES, K.; LI, W.; SU, W.; WISCOMBE, W. Improving the description of sunglint for accurate prediction of remotely sensed radiances. J Quant Spectrosc Radiat Transfer, v. 109, n. 14, p. 2364-2375, 2008.

PAN, X.; WONG, G.T.; SHIAH, F.K.; HO, T.Y. Enhancement of biological productivity by internal waves: observations in the summertime in the northern South China Sea. J Oceanogr, v. 68, n. 3, p. 427-437, 2012.

PELLON DE MIRANDA, F.; MARMOL, A.M.Q.; PEDROSO, E.C.; BEISL, C.H.; WELGAN, P.; MORALES, L.M. Analysis of RADARSAT-1 data for offshore monitoring activities in the Cantarell Complex, Gulf of Mexico, using the unsupervised semivariogram textural classifier (USTC). Can J Remote Sens, v. 30, n. 3, p. 424-436, 2004.

PINGREE, R.D.; NEW, A.L. Structure, seasonal development and sunglint spatial coherence of the internal tide on the Celtic and Armorican shelves and in the Bay of Biscay. Deep-Sea Res Pt I, v. 42, n. 2, p. 245-284, 1995.

PISANO, A.; BIGNAMI, F.; SANTOLERI, R. Oil spill detection in glint-contaminated near-Infrared MODIS imagery. Remote Sens, v. 7, n. 1, p. 1112-1134, 2015.
ROBINSON, I.S. Measuring the oceans from space: the principles and methods of satellite oceanography. Berlin: Springer, 2004

RODRÍGUEZ, J.M.; BARTON, E.D.; HERNÁNDEZ-LEÓN, S.; ARÍSTEGUI, J. The influence of mesoscale physical processes on the larval fish community in the Canaries CTZ, in summer. Prog Oceanogr, v. 62, n. 2, p. 171-188, 2004.

SCHONTEN, M.W.; RUIJTER, W.P.; LEEUWEN, P.J.; LUTJEHARMS, J.R. Translation, decay and splitting of Agulhas rings in the development. J Geophys Res, v. 105, n. 15, p. 21913-21925, 2000.

SIGNORINI, S.R. On the circulation and the volume transport of the Brazil current between the Cape of São Tomé and Guanabara Bay. Deep-Sea Res, v. 25, n. 5, p. 481-490, 1978.

SOULES, S.D. Sun glitter viewd from space. Deep Sea Res Oceanogr Abstr, v. 17, n. 1, p. 191-195, 1970.

SOUTELINO, R.G.; DA SILVEIRA, I.C.A.; GANGOPADHYAY, A.A.M.J.; MIRANDA, J.A. Is the Brazil current eddy-dominated to the north of $20^{\circ} \mathrm{S}$ ? Geophys Res Lett, v. 38, n. 3, L03607, 2011.

STEINMETZ, F.; DESCHAMPS, P.-Y.; RAMON, D. Atmospheric correction in presence of sun glint: application to MERIS. Opt Express, v. 19, n. 10, p.9783-9800, 2011.

STRAMMA, L.; ENGLAND, M. On the water masses and mean circulation of the South Atlantic Ocean. J Geophys Res, v. 104, n. C9, p. 20863-20883, 1999.

TANRÉ, D.; HERMAN, M.; DESCHAMPS, P.Y.; DE LEFFE, A. Atmospheric modeling for space measurements of ground reflectances, including bidirectional properties. Appl Optics, v. 18, p. 3587-3594, 1979.

THUILLIER, G.; HERSÉ, M.; FOUJOLS, T.; PEETERMANS, W.; GILLOTAY, D.; SIMON, P.C.; MANDEL, H. The solar spectral irradiance from 200 to $2400 \mathrm{~nm}$ as measured by the SOLSPEC spectrometer from the ATLAS and EURECA missions. Sol Phys, v. 214, n. 1, p. 1-22, 2003.

TOPOUZELIS, K.; KITSIOU, D. Detection and classification of mesoscale atmospheric phenomena above sea in SAR imagery. Remote Sens Environ, v. 160, p. 263-272, 2015.

VACHON, P.M.; JOHANNESSEN, O. M.; JOHANNESSEN, J.A. An ERS 1 synthetic aperture radar image of atmospheric lee waves. J Geophys Res, v. 99, n. C11, p. 2248222490, 1994.

VIOLETTE, P.E.; PETEHERYCH, S.; GOWER, J. F. R. Oceanographic implications of features in NOAA satellite visible imagery. Bound-Lay Meteorol, v. 18, n. 2, p. 159-175, 1980.

WALD, L.; MONGET, J.M. Sea surface winds from sun glitter observations. J Geophys Res, v. 88, n. C4, p. 2547-2555, 1983.

WANG, M.; BAILEY, S.W. Correction of the sunglint contamination on the SeaWiFS aerosol optical thickness retrievals. In: McCLAIN, C. R. et al. (Eds.). SeaWiFS postlaunch calibration and validation analyses. Washington: NASA, 2000. (NASA Technical Memorandum, v. 9). p. 65-69.

WANG, M.; BAILEY, S.W. Correction of sun glint contamination on the SeaWiFS ocean and atmosphere products. Appl Optics, v. 40, n. 27, p. 4790-4798, 2001.

WURTEle, G.; SHAMAN, R.D. Atmospheric lee waves. Annu Rev Fluid Mech, v.28, p.429-476, 1996. 
WYLIE, D.P.; HINTON, B.B.; MILLETT, K. M.A Comparison of three satellite-based methods for estimating surface winds over oceans. J Appl Meteorol, v. 20, n. 4, p. 439-449, 1981.

THUILLIER, G.; HERSÉ, M.; FOUJOLS, T.; PEETERMANS, W.; GILLOTAY, D.; SIMON, P.C.; MANDEL, H. A line in the sea. Nature, v. 371, p. 689-692, 1994.

YOUNG, G.S.; KRISTOVICH, D.A.; HJELMFELT, M.R.; FOSTER, R.C. Supplement to rolls, streets, waves, and more. Bull Am Meteorol Soc, v. 83, n. 7, p. 1001-1001, 2002.

ZEISSE, C.R. Radiance of the ocean horizon. J Opt Soc Am A Opt Image Sci Vis, v. 12, n. 9, p. 2022-2030, 1995.
ZHANG, H.; WANG, M. Evaluation of sun glint models using MODIS measurements. J Quant Spectrosc Radiat Transfer, v. 111, n. 3, p. 492-506, 2010.

ZHENG, Q.; YAN, X.-H.; KLEMAS, V. Statistical and dynamical analysis of internal waves on the continental shelf of the middle Atlantic bight from space shuttle photographs. J Geophys Res, v. 98, n. C5, p. 8495-8504, 1993.

\section{Fontes da Internet}

Oceancolor da Nasa: http://seadas.gsfc.nasa.gov CPTEC/INPE: http://satelite.cptec.inpe.br/oceano/ AVISO: http://www.aviso.altimetry.fr/duacs/

This is an Open Access article distributed under the terms of the Creative Commons Attribution Non-Commercial License which permits unrestricted non-commercial use, distribution, and reproduction in any medium provided the original work is properly cited. 